\title{
SMC Complexes Are Guarded by the SUMO Protease Ulp2 Against SUMO-Chain-Mediated Turnover
}

\author{
Ivan Psakhye ${ }^{1}$ and Dana Branzei ${ }^{1,2 *}$ \\ ${ }^{1}$ IFOM, the FIRC Institute of Molecular Oncology, Via Adamello 16, 20139 Milan, \\ Italy \\ ${ }^{2}$ Istituto di Genetica Molecolare, Consiglio Nazionale delle Ricerche (IGM-CNR), \\ Via Abbiategrasso 207, 27100 Pavia, Italy \\ * To whom correspondence should be addressed. Tel: +39-02-574303259; Fax: +39- \\ 02-574303231; Email: dana.branzei@ifom.eu
}

\section{Keywords}

Cohesin, Condensin, Smc5/6, SMC complexes, Pds5, SUMO, SENP/Ulp proteases, SUMO chains, STUbL-mediated proteasomal degradation, Slx $5 / 8$ 


\section{ABSTRACT}

Structural maintenance of chromosomes (SMC) complexes, cohesin, condensin and Smc5/6, are essential for viability and participate in multiple processes, including sister chromatid cohesion, chromosome condensation, and DNA repair. Here we show that SUMO chains target all three SMC complexes and are antagonized by the SUMO protease Ulp2 to prevent their turnover. We uncover that the essential role of the cohesin-associated subunit Pds5 is to counteract SUMO chains jointly with Ulp2. Importantly, fusion of Ulp2 to kleisin Scc1 supports viability of PDS5 null cells and protects cohesin from proteasomal degradation mediated by the SUMO-targeted ubiquitin ligase Slx5/S1x8. The lethality of PDS5 deleted cells can also be bypassed by simultaneous loss of the PCNA unloader, Elg1, and the cohesin releaser, Wpl1, but only when Ulp2 is functional. Condensin and Smc5/6 complex are similarly guarded by Ulp2 against unscheduled SUMO-chain assembly, which we propose to time the availability of SMC complexes on chromatin. 


\section{INTRODUCTION}

Posttranslational modification of proteins with the small ubiquitin-like modifier (SUMO) is essential for eukaryotic cells as it regulates substrate fate by affecting protein interactions, activity, localization and abundance (1). SUMOylation frequently targets entire protein groups, actively engaged in common functions $(2,3)$, and fosters protein complex formation through SUMO binding to SUMO-interacting motifs (SIMs). Similar to ubiquitin, SUMO is conjugated to exposed lysines on the substrates leading to monoSUMOylation or, if several lysines are modified, to multiSUMOylation. Moreover, mono/multiSUMOylation may be extended to SUMO chains when lysines of SUMO conjugated to the substrate are being further modified with SUMO, leading to substrate polySUMOylation. The modifications are reversible and counteracted by SUMO proteases, which have different localization, substrate and SUMO-linkage specificities (4). In budding yeast, the SUMO protease Ulp2 has preference for SUMO chains and prevents substrate polySUMOylation, which can be further recognized by ubiquitin E3 ligases containing multiple SIMs. These so-called SUMO-targeted ubiquitin ligases (STUbLs) can mediate proteolytic or non-proteolytic ubiquitylation of the SUMOylated substrate (5). Thus, SUMO chains disassembled by the Ulp2 protease may function as a countdown timer if they are assembled on the substrates of STUbLs.

We recently reported that SUMO-chain/Ulp2-protease-regulated proteasomal degradation is a mechanism that times the availability of DDK, a key replication initiation regulator (6). To extend our findings beyond DNA replication, we performed an unbiased SILAC-based proteomic screen to uncover degradation-prone SUMO conjugates that decrease in abundance in the absence of Ulp2 specifically in a SUMOchain-dependent manner. Strikingly, we found subunits of all three SMC complexes, 
cohesin, condensin and Smc5/6 $(7,8)$ as potential hits, suggesting that the abundance of SMC complexes is regulated via a SUMO-chain-dependent mechanism.

Cohesin was previously shown to become SUMOylated upon loading onto DNA, and loss of cohesin SUMOylation resulted in cohesion defects $(9,10)$. Moreover, the cohesin associated factor, Pds5, was proposed to protect monoSUMOylated cohesin and facilitate cohesion by preventing via a yet unidentified mechanism Siz2 SUMOligase-mediated cohesin polySUMOylation that leads to increased Slx5/8 STUbLmediated proteasome degradation of the cohesin kleisin, Scc1 (11). More recently, depletion of SENP6, the human ortholog of Ulp2, was shown to decrease cohesin binding to chromatin and cause cohesion defects (12). Taken together, these data suggest that mono/multiSUMOylation of DNA-loaded cohesin is important for cohesion, whereas polySUMOylation induced in $p d s 5$ mutants targets cohesin for STUbL-mediated proteasomal turnover compromising cohesion.

Here we aimed to address if and how Ulp2 protects cohesin and other SMC complexes from SUMO-chain-targeted turnover. We find that fusion of Ulp2 to the cohesin's kleisin Scc1 protects cohesin from proteasomal degradation and supports viability in the complete absence of Pds5 that is otherwise essential. Moreover, we identify that simultaneous loss of the PCNA unloader, Elg1, and the cohesin releaser, Wp11, allows viability of PDS5 null cells in a manner strictly depending on Ulp2 function. These results indicate that the essential function of Pds5 is to counteract SUMO chains jointly with Ulp2 rather than support cohesin activity in other direct ways. Condensin and Smc5/6 are also safeguarded by Ulp2 against unscheduled SUMO chain assembly, overall suggesting a SUMO-chain/Ulp2-protease-governed mechanism that instructs SMC complexes availability on chromatin. 


\section{MATERIAL AND METHODS}

\section{Yeast Strains}

Chromosomally tagged Saccharomyces cerevisiae strains and mutants were constructed by a PCR-based strategy, by genetic crosses and standard techniques (13). Standard cloning and site-directed mutagenesis techniques were used. Strains and all genetic manipulations were verified by polymerase chain reaction (PCR), sequencing and phenotype. All yeast strains used in this work except those specifically indicated and used for the yeast two-hybrid (Y2H) studies are isogenic to W303 background and are listed in the Table 1.

Table 1. Saccharomyces cerevisiae strains used in this study.

\begin{tabular}{|c|c|c|}
\hline Strain & Relevant Genotype & Reference \\
\hline $\begin{array}{l}\text { FY1363 } \\
(\mathrm{W} 303 \\
\text { WT) }\end{array}$ & MATa ade2-1 can1-100 his3-11-15 leu2-3,112 trp1-1 ura3-1 RAD5+ & $\begin{array}{l}\text { Lab } \\
\text { collection }\end{array}$ \\
\hline $\begin{array}{l}\text { FY1364 } \\
(\text { W303 } \\
\text { WT) }\end{array}$ & MATalpha ade2-1 can1-100 his3-11-15 leu2-3,112 trp1-1 ura3-1 RAD5+ & $\begin{array}{l}\text { Lab } \\
\text { collection }\end{array}$ \\
\hline HY0752 & MATa ulp2::KANMX4 & This study \\
\hline HY9633 & $\begin{array}{c}\text { MATa cim3-1(URA3) smt3::HIS3MX6 lys } 1:: \text { KanMX4 arg4::HphNT1 ura3- } \\
\text { 52::pADH1-7His-SMT3-tADH1(URA3) ulp2::NatMX4 }\end{array}$ & This study \\
\hline HY9636 & $\begin{array}{c}\text { MATa cim3-1(URA3) smt } 3: \because H I S 3 M X 6 \text { lys } 1: \because \text { KanMX4 arg4 }:: H p h N T 1 \text { ura3- } \\
\text { 52::pADH1-7His-smt3-KRall-tADH1(URA3) ulp2::NatMX4 }\end{array}$ & This study \\
\hline Y2HGold & 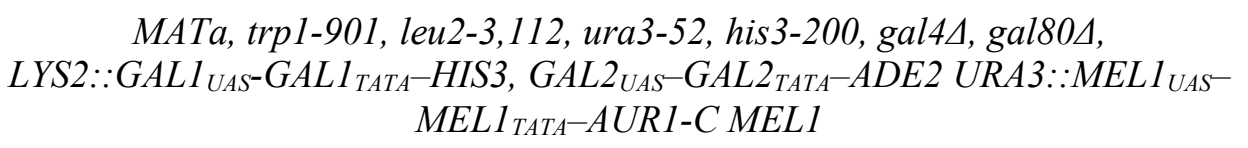 & Takara \\
\hline HY9925 & MATa ULP2-9PK::HIS3MX6 PDS5-13MYC::TRP1 & This study \\
\hline HY5354 & MATa (TRP1)pSMT3-7His-SMT3 & This study \\
\hline HY5406 & MATalpha (TRP1)pSMT3-7His-SMT3 & This study \\
\hline HY9933 & MATalpha PDS5-3HA::HIS3MX6 & This study \\
\hline HY9934 & MATa (TRP1)pSMT3-7His-SMT3 PDS5-3HA::HIS3MX6 & This study \\
\hline
\end{tabular}




\begin{tabular}{|c|c|c|}
\hline HY9918 & MATa/MATalpha ELG1/elg1::HIS3MX6 WPL1/wpl1::TRP1 & This study \\
\hline HY10012 & $\begin{array}{l}\text { MATa/MATalpha smt } 3:: H I S 3 M X 6 / \text { smt } 3:: H I S 3 M X 6 \text { ura3-52::pADH1-7His- } \\
\text { smt3-KRall-tADH1(URA3)/ura3-52::pADH1-7His-smt3-KRall-tADH1(URA3) } \\
\text { ELG1/elg1::KanMX4 WPL1/wpl1::TRP1 PDS5/pds5::NatMX4 }\end{array}$ & This study \\
\hline HY9927 & $\begin{array}{c}\text { MATa/MATalpha ELG1/elg1::HIS3MX6 WPL1/wpl1::TRP1 } \\
\text { PDS5/pds5::NatMX4 }\end{array}$ & This study \\
\hline HY1978 & MATalpha elg1::HIS3MX6 & $\begin{array}{l}\text { Lab } \\
\text { collection }\end{array}$ \\
\hline HY6507 & MATa wpll::TRP1 & $\begin{array}{l}\text { Lab } \\
\text { collection }\end{array}$ \\
\hline HY9940 & MATa elg1::HIS3MX6 wpl1::TRP1 & This study \\
\hline HY9941 & MATalpha elg1::HIS3MX6 wpl1::TRP1 & This study \\
\hline HY9978 & MATa elg1::HIS3MX6 wpll::TRP1 pds5::NatMX4 & This study \\
\hline HY10053 & $\begin{array}{c}\text { MATa smt3::HIS3MX6 ura3-52::pADH1-7His-smt3-KRall-tADH1(URA3) } \\
\text { pds5::NatMX4 }\end{array}$ & This study \\
\hline HY 10060 & $\begin{aligned} \text { MATalpha smt } 3: & \text { HIS3MX6 ura3-52::pADH1-7His-smt3-KRall-tADH1(URA3) } \\
& \text { elg1::KanMX4 wpl1::TRP1 pds5::NatMX4 }\end{aligned}$ & This study \\
\hline HY8053 & MATa smt3::HIS3MX6 ura3-52::pADH1-7His-smt3-KRall-tADH1(URA3) & This study \\
\hline HY10335 & $\begin{array}{c}\text { MATa/MATalpha smt } 3:: H I S 3 M X 6 / \text { smt3::HIS3MX6 ura3-52::pADH1-7His- } \\
\text { smt3-KRall-tADH1(URA3)/ura3-52::pADH1-7His-smt3-KRall-tADH1(URA3) } \\
\text { ELG1/elg1::KanMX4 WPL1/wpl1::TRP1 SCC3/scc3::NatMX4 }\end{array}$ & This study \\
\hline HY 10272 & $\begin{array}{l}\text { MATa/MATalpha smt } 3:: H I S 3 M X 6 / \text { smt3::HIS3MX6 ura3-52::pADH1-7His- } \\
\text { smt3-KRall-tADH1(URA3)/ura3-52::pADH1-7His-smt3-KRall-tADH1(URA3) } \\
\text { ELG1/elg1::KanMX4 WPL1/wpl1::TRP1 ECO1/eco1::NatMX4 }\end{array}$ & This study \\
\hline HY10111 & MATalpha elg1::HIS3MX6 wpl1::TRP1 (KanMX4)pGALS-ULP2 & This study \\
\hline HY10114 & MATa elg1::HIS3MX6 wpl1::TRP1 pds5::NatMX4 (KanMX4)pGALS-ULP2 & This study \\
\hline HY10115 & $\begin{array}{c}\text { MATa elg1::HIS3MX6 wpl1: }: \text { TRP1 pds5::NatMX4 (KanMX4)pGALS-ULP2 } \\
\text { ulp1-C376(URA3) }\end{array}$ & This study \\
\hline HY10117 & $\begin{array}{c}\text { MATalpha elg1::HIS3MX6 wpl1::TRP1 (KanMX4)pGALS-ULP2 } \\
\text { ulp1-C376(URA3) }\end{array}$ & This study \\
\hline HY5256 & MATa ulp2::KANMX4 HU sensitivity spontaneous suppressor \#1 & This study \\
\hline HY5257 & MATa ulp2::KANMX4 HU sensitivity spontaneous suppressor \#2 & This study \\
\hline HY5258 & MATa ulp2::KANMX4 HU sensitivity spontaneous suppressor \#3 & This study \\
\hline HY5259 & MATa ulp2::KANMX4 HU sensitivity spontaneous suppressor \#4 & This study \\
\hline HY5260 & MATa ulp2::KANMX4 HU sensitivity spontaneous suppressor \#5 & This study \\
\hline HY5261 & MATa ulp2::KANMX4 HU sensitivity spontaneous suppressor \#3 backcrossed & This study \\
\hline
\end{tabular}




\begin{tabular}{|c|c|c|}
\hline HY5262 & MATa ulp2::KANMX4 HU sensitivity spontaneous suppressor \#5 backcrossed & This study \\
\hline HY5335 & MATa ulp2::KANMX4 HU sensitivity spontaneous suppressor \#1 backcrossed & This study \\
\hline HY5340 & MATa ulp2::KANMX4 HU sensitivity spontaneous suppressor \#2 backcrossed & This study \\
\hline HY5341 & MATa ulp2::KANMX4 HU sensitivity spontaneous suppressor \#4 backcrossed & This study \\
\hline HY5630 & $\begin{array}{l}\text { MATa/MATalpha ULP1/ulp1::hphNT1 ULP2/ulp2::HIS3MX6 } \\
\text { LYS1/lys1::NatMX4 SMT3/(TRP1)pSMT3-7His-SMT3 }\end{array}$ & This study \\
\hline HY5631 & $\begin{array}{l}\text { MATa/MATalpha ULP1/ulp1-N253::KANMX6 ULP2/ulp2::HIS3MX6 } \\
\text { LYS1/lys1::NatMX4 SMT3/(TRP1)pSMT3-7His-SMT3 ARG4/arg4::hphNT1 }\end{array}$ & This study \\
\hline HY5908 & $\begin{array}{l}\text { MATa/MATalpha ULP1/ulp1-sup(URA3) ULP2/ulp2::HIS3MX6 } \\
\text { LYS1/lys1::NatMX4 SMT3/(TRP1)pSMT3-7His-SMT3 }\end{array}$ & $\begin{array}{c}\text { This } \\
\text { study; } \\
\text { HY5630 } \\
\text { derivative }\end{array}$ \\
\hline HY5844 & $\begin{array}{c}\text { MATa/MATalpha ULP1/ulp1-C376(URA3) ULP2/ulp2::HIS3MX6 } \\
\text { LYS1/lys1::NatMX4 SMT3/(TRP1)pSMT3-7His-SMT3 }\end{array}$ & $\begin{array}{c}\text { This } \\
\text { study; } \\
\text { HY5630 } \\
\text { derivative }\end{array}$ \\
\hline HY5894 & MATalpha ulp1-sup(URA3) & This study \\
\hline HY5896 & MATalpha ulp1-sup(URA3) ulp2::HIS3MX6 & This study \\
\hline HY5902 & MATalpha ulp1-C376(URA3) & This study \\
\hline HY5904 & MATalpha ulp1-C376(URA3) ulp2::HIS3MX6 & This study \\
\hline HY5834 & MATa (TRP1)pSMT3-7His-SMT3 ULP1-6HA::hphNT1 & This study \\
\hline HY5835 & MATa ulp1-sup-6HA::hphNT1 ulp2::KANMX4 & $\begin{array}{c}\text { This } \\
\text { study; } \\
\text { HY5262 } \\
\text { derivative }\end{array}$ \\
\hline HY10219 & $\begin{array}{c}\text { MATa cim3-1(URA3) (TRP1)pSMT3-7His-SMT3 SCC1-6HA::HphNT1 } \\
\text { (natNT2)GALS-3HA-PDS5 }\end{array}$ & This study \\
\hline HY10232 & $\begin{array}{c}\text { MATa cim3-1(URA3) (TRP1)pSMT3-7His-SMT3 SCC1-6HA::HphNT1 } \\
\text { (natNT2)GALS-3HA-PDS5 ulp2::KANMX4 }\end{array}$ & This study \\
\hline HY10233 & $\begin{array}{c}\text { MATa cim3-1(URA3) (TRP1)pSMT3-7His-SMT3 SCC1-6HA::HphNT1 } \\
\text { (natNT2)GALS-3HA-PDS5 ulp2::KANMX4 }\end{array}$ & This study \\
\hline HY10247 & $\begin{array}{l}\text { MATa cim3-1(URA3) (TRP1)pSMT3-7His-SMT3 SCC1-6HA::HphNT1 } \\
\text { (natNT2)GALS-3HA-PDS5 ulp2::KANMX4 slx5::HIS3MX6 }\end{array}$ & This study \\
\hline HY10248 & $\begin{array}{c}\text { MATa cim3-1(URA3) (TRP1)pSMT3-7His-SMT3 SCC1-6HA::HphNT1 } \\
\text { (natNT2)GALS-3HA-PDS5 ulp2::KANMX4 slx5::HIS3MX6 }\end{array}$ & This study \\
\hline HY10204 & $\begin{array}{c}\text { MATa SCC1-6HA::HphNT1 (TRP1)pSMT3-7His-SMT3 } \\
\text { (natNT2)GALS-3HA-PDS5 }\end{array}$ & This study \\
\hline
\end{tabular}




\begin{tabular}{|c|c|c|}
\hline HY10205 & $\begin{array}{l}\text { MATa SCC1-6HA::HphNT1 (TRP1)pSMT3-7His-SMT3 } \\
\text { (natNT2)GALS-3HA-PDS5 sgs1 ::HIS3MX6 }\end{array}$ & This study \\
\hline HY10336 & $\begin{aligned} & \text { MATa smt3:: } \text { HIS3MX6 ura3-52::pADH1-7His-smt3-KRall-tADH1(URA3) } \\
& \text { SCC1-6HA }: \because H p h N 1 \text { (natNT2)GALS-3HA-PDS5 }\end{aligned}$ & This study \\
\hline HY10338 & $\begin{array}{l}\text { MATa smt3::HIS3MX6 ura3-52::pADH1-7His-smt3-KRall-tADH1(URA3) } \\
\text { SCC1-6HA::HphNT1 (natNT2)GALS-3HA-PDS5 ulp2::KANMX4 }\end{array}$ & This study \\
\hline HY10725 & $\begin{array}{l}\text { MATa smt3::HIS3MX6 ura3-52::pADH1-7His-smt3-KRall-tADH1(URA3) } \\
\text { SCC1-6HA::HphNT1 (natNT2)GALS-3HA-PDS5 ulp2-C624S-9PK::HIS3MX6 }\end{array}$ & This study \\
\hline HY10072 & MATa (TRP1)pSMT3-7His-SMT3 PDS5-3HA::HIS3MX6 SCC1-6HA::HphNT1 & This study \\
\hline HY10073 & $\begin{array}{c}\text { MATa (TRP1)pSMT3-7His-SMT3 PDS5-3HA ::HIS3MX6 SCC1-6HA::HphNT1 } \\
\text { ulp2::NatMX4 }\end{array}$ & This study \\
\hline HY10246 & $\begin{array}{c}\text { MATa elg1::HIS3MX6 wpll::TRP1 pds5::NatMX4 (KanMX4)pGALS-ULP2 } \\
\text { scc1-ulp2 }{ }_{734^{-} \text {C624S-6HA::HphNT1 }}\end{array}$ & This study \\
\hline HY10344 & $\begin{array}{l}\text { MATalpha elg1::HIS3MX6 wpll::TRP1 pds5::NatMX4 } \\
\text { (KanMX4)pGALS-ULP2 scc1-ulp2 } 2_{734-6 H A:: H p h N T 1}\end{array}$ & This study \\
\hline HY10473 & MATa (natNT2) GALS-3HA-PDS5 sgs $1: \because H I S 3 M X 6$ & This study \\
\hline HY10475 & MATa wpl1::TRP1 (natNT2)GALS-3HA-PDS5 scc1-ulp2 ${ }_{734-6 H A:: H p h N T 1}$ & This study \\
\hline HY10479 & 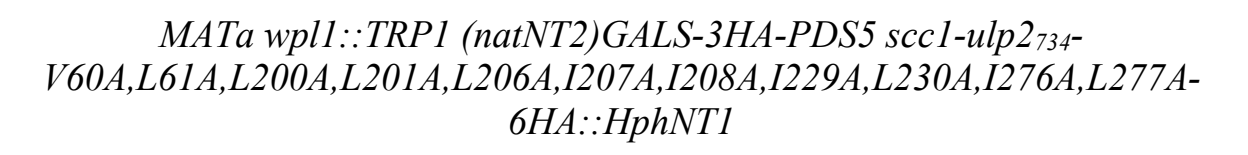 & This study \\
\hline HY10480 & $\begin{array}{c}\text { MATa wpl1::TRP1 (natNT2)GALS-3HA-PDS5 sgs } 1: \because H I S 3 M X 6 \\
\text { scc1-ulp2 }{ }_{734-C 624 S-6 H A:: H p h N T 1}\end{array}$ & This study \\
\hline HY10379 & $\begin{array}{c}\text { MATa wpl1::TRP1 elg1 :HHS3MX6 pds5::NatMX4 } \\
\text { ura3-52::pADH1-7His-SMT3-tADH1(URA3) (KanMX4)pGALS-ULP2 } \\
\text { scc1-ulp2 }{ }_{734-C 624 S-6 H A: \because H p h N T 1}\end{array}$ & This study \\
\hline HY10478 & $\begin{array}{c}\text { MATalpha wpl1::TRP1 elg1::HIS3MX6 pds5::NatMX4 } \\
\text { ura3-52::pADH1-7His-SMT3-tADH1(URA3) (KanMX4)pGALS-ULP2 } \\
\text { scc1-ulp2 }{ }_{734-6 H A:: H p h N T 1}\end{array}$ & This study \\
\hline HY 10220 & MATalpha wpl1::TRP1 elg1::HIS3MX6 scc1-ulp2 ${ }_{734-6 H A:: H p h N T 1}$ & This study \\
\hline HY10203 & MATalpha wpl1::TRP1 elg1::HIS3MX6 scc1-ulp2 ${ }_{734-C 624 S-6 H A:: H p h N T 1}$ & This study \\
\hline HY10202 & $\begin{array}{c}\text { MATalpha wpl1::TRP1 elg1::HIS3MX6 scc1-ulp2 } 7_{734^{-}} \\
\text {V60A,L61A,L200A,L201A,L206A,I207A,I208A,I229A,L230A,I276A,L277A- } \\
\text { 6HA::HphNT1 }\end{array}$ & This study \\
\hline HY10570 & MATa ADE2 HIS3 & This study \\
\hline HY 10567 & MATa ADE2 HIS3 smc2-8-6HA::HphNT1 & This study \\
\hline HY10568 & MATalpha ADE2 HIS3 ulp2::KANMX4 & This study \\
\hline HY10569 & MATa ADE2 HIS3 smc2-8-6HA::HphNT1 ulp2::KANMX4 & This study \\
\hline
\end{tabular}




\begin{tabular}{|c|c|c|}
\hline HY10601 & $\begin{array}{c}\text { MATa ADE2 smc2-8-6HA::HphNT1 ulp2::KANMX4 smt3::HIS3MX6 } \\
\text { ura3-52::pADH1-7His-smt3-KRall-tADH1(URA3) }\end{array}$ & This study \\
\hline HY10602 & $\begin{array}{l}\text { MATalpha ADE2 smc2-8-6HA::HphNT1 smt3::HIS3MX6 } \\
\text { ura3-52::pADH1-7His-smt3-KRall-tADH1(URA3) }\end{array}$ & This study \\
\hline HY10599 & MATa ADE2 smc2-8-6HA::HphNT1 slx5::NatMX4 ulp2::HIS3MX6 & This study \\
\hline HY10565 & MATa ADE2 smc2-8-6HA::HphNT1 slx5::NatMX4 HIS3 & This study \\
\hline HY10564 & MATa ADE2 slx $5: \because$ NatMX4 ulp $2: \because H I S 3 M X 6$ & This study \\
\hline HY10566 & MATa ADE2 slx5::NatMX4 HIS3 & This study \\
\hline HY 10600 & MATa ADE2 smc2-8-6HA::HphNT1 slx5::NatMX4 ulp2::HIS3MX6 & This study \\
\hline HY10689 & MATa (TRP1)pSMT3-7His-SMT3 BRN1-6HA::HphNT1 & This study \\
\hline HY10697 & MATa (TRP1)pSMT3-7His-SMT3 BRN1-6HA::HphNT1 ulp2::NatMX4 & This study \\
\hline HY10691 & $\begin{array}{c}\text { MATa smt3::HIS3MX6 ura3-52::pADH1-7His-smt3-KRall-tADH1(URA3) } \\
\text { BRN1-6HA::HphNT1 }\end{array}$ & This study \\
\hline HY10699 & $\begin{array}{c}\text { MATalpha smt3::HIS3MX6 ura3-52::pADH1-7His-smt3-KRall-tADH1(URA3) } \\
\text { BRN1-6HA:HphNT1 ulp2:NatMX4 }\end{array}$ & This study \\
\hline HY10234 & MATa (KanMX6)pGAL1-3HA-YCS4 & This study \\
\hline HY10237 & MATalpha (KanMX6)pGAL1-3HA-YCG1 & This study \\
\hline HY10236 & $\begin{array}{c}\text { MATalpha smt3::HIS3MX6 ura3-52::pADH1-7His-smt3-KRall-tADH1(URA3) } \\
\text { (KanMX6)pGAL1-3HA-YCS4 }\end{array}$ & This study \\
\hline HY10238 & $\begin{array}{c}\text { MATa smt3::HIS3MX6 ura3-52::pADH1-7His-smt3-KRall-tADH1(URA3) } \\
\text { (KanMX6)pGAL1-3HA-YCG1 }\end{array}$ & This study \\
\hline HY10342 & $\begin{array}{c}\text { MATalpha smt3::HIS3MX6 ura3-52::pADH1-7His-smt3-KRall-tADH1(URA3) } \\
\text { (KanMX6)pGAL1-3HA-YCS4 BRN1-6HA:HphNT1 }\end{array}$ & This study \\
\hline HY10343 & $\begin{array}{c}\text { MATa smt3::HIS3MX6 ura3-52::pADH1-7His-smt3-KRall-tADH1(URA3) } \\
\text { (KanMX6)pGAL1-3HA-YCG1 BRN1-6HA::HphNT1 }\end{array}$ & This study \\
\hline HY10742 & MATa (TRP1)pSMT3-7His-SMT3 BRN1-6HA::HphNT1 ulp2::HIS3MX6 & This study \\
\hline HY10748 & MATa (TRP1)pSMT3-7His-SMT3 BRN1-6HA::HphNT1 cim3-1(URA3) & This study \\
\hline HY10750 & $\begin{array}{c}\text { MATa (TRP1)pSMT3-7His-SMT3 BRN1-6HA::HphNT1 cim3-1(URA3) } \\
\text { ulp2::HIS3MX6 }\end{array}$ & This study \\
\hline HY10397 & MATalpha smc6-56-13myc::KanMX & This study \\
\hline HY5156 & MATalpha ulp2::HIS3MX6 & This study \\
\hline HY10612 & MATa ulp2::HIS3MX6 smc6-56-13myc::KanMX & This study \\
\hline HY10681 & MATa siz2::HphNT1 smc6-56-13myc::KanMX & This study \\
\hline HY10683 & MATa siz2::HphNT1 ulp2::HIS3MX6 smc6-56-13myc::KanMX & This study \\
\hline
\end{tabular}




\begin{tabular}{|c|c|c|}
\hline HY6650 & MATalpha siz2::HphNT1 ulp2::HIS3MX6 & This study \\
\hline HY6171 & MATa siz2::HphNT1 & This study \\
\hline HY10780 & MATa (TRP1)pSMT3-7His-SMT3 NSE4-6HA::NatNT2 cim3-1(URA3) & This study \\
\hline HY10782 & $\begin{array}{c}\text { MATa (TRP1)pSMT3-7His-SMT3 NSE4-6HA::NatNT2 cim3-1(URA3) } \\
\text { ulp2::HIS3MX6 }\end{array}$ & This study \\
\hline HY10784 & $\begin{array}{c}\text { MATalpha (TRP1)pSMT3-7His-SMT3 NSE4-6HA::NatNT2 cim3-1(URA3) } \\
\text { slx5::KANMX4 }\end{array}$ & This study \\
\hline HY10773 & MATalpha (TRP1)pSMT3-7His-SMT3 NSE4-6HA::NatNT2 & This study \\
\hline HY10774 & MATa (TRP1)pSMT3-7His-SMT3 NSE4-6HA::NatNT2 ulp2::HIS3MX6 & This study \\
\hline HY10794 & $\begin{array}{c}\text { MATa smt3::HIS3MX6 ura3-52::pADH1-7His-smt3-KRall-tADH1(URA3) } \\
\text { NSE4-6HA::NatNT2 }\end{array}$ & This study \\
\hline HY10796 & $\begin{array}{c}\text { MATa smt3::HIS3MX6 ura3-52::pADH1-7His-smt3-KRall-tADH1(URA3) } \\
\text { NSE4-6HA::NatNT2 ulp2::KANMX4 }\end{array}$ & This study \\
\hline HY8072 & MATalpha smt3::HIS3MX6 ura3-52::pADH1-7His-smt3-KRall-tADH1(URA3) & This study \\
\hline HY10398 & $\begin{array}{c}\text { MATa smt3::HIS3MX6 ura3-52::pADH1-7His-smt3-KRall-tADH1(URA3) } \\
\text { smc6-56-13myc::KanMX }\end{array}$ & This study \\
\hline HY10273 & MATalpha nse3-ts-12-9myc::TRP1 & This study \\
\hline HY10275 & $\begin{array}{c}\text { MATalpha smt3::HIS3MX6 ura3-52::pADH1-7His-smt3-KRall-tADH1(URA3) } \\
\text { nse3-ts-12-9myc::TRP1 }\end{array}$ & This study \\
\hline HY10283 & MATa leu2::GPD1-OsTIR1(LEU2) NSE5-AID::KanMX NSE4-9PK::HIS3MX6 & This study \\
\hline HY10370 & $\begin{array}{c}\text { MATa smt3::HIS3MX6 ura3-52::pADH1-7His-smt3-KRall-tADH1(URA3) } \\
\text { leu2::GPD1-OsTIR1(LEU2) }\end{array}$ & This study \\
\hline HY10374 & $\begin{array}{c}\text { MATa smt3::HIS3MX6 ura3-52::pADH1-7His-smt3-KRall-tADH1(URA3) } \\
\text { leu2 }: \because \text { GPD1-OsTIR1(LEU2) NSE5-AID ::KanMX }\end{array}$ & This study \\
\hline HY10376 & $\begin{array}{l}\text { MATa smt3::HIS3MX6 ura3-52::pADH1-7His-smt3-KRall-tADH1(URA3) } \\
\text { leu2::GPD1-OsTIR1(LEU2) NSE5-AID::KanMX NSE4-9PK::HIS3MX6 }\end{array}$ & This study \\
\hline HY6969 & MATa slx $5:: N a t M X 4$ & This study \\
\hline HY10400 & MATalpha smc6-P4-13myc::KanMX & This study \\
\hline HY10618 & MATa ulp2::HIS3MX6 smc6-P4-13myc::KanMX & This study \\
\hline HY10622 & MATa ulp2::HIS3MX6 smc6-P4-13myc::KanMX slx5::NatMX4 & This study \\
\hline HY10620 & MATa smc6-P4-13myc $:$ KanMX slx $5: \because N a t M X 4$ & This study \\
\hline HY10687 & MATa ulp2::HIS3MX6 smc6-P4-13myc::KanMX siz2::HphNT1 & This study \\
\hline HY10685 & MATa smc6-P4-13myc ::KanMX siz2 $\because: H p h N T 1$ & This study \\
\hline HY6258 & MATa/MATalpha ULP2/ulp2::HIS3MX6 ESC2/esc2::KanMX4 & This study \\
\hline
\end{tabular}




\begin{tabular}{|c|c|c|}
\hline HY6256 & MATa/MATalpha ULP2/ulp2::HIS3MX6 SGS1/sgs1::NatMX4 & This study \\
\hline HY6257 & MATa/MATalpha ULP2/ulp2::KANMX4 RRM3/rrm3::HIS3MX6 & This study \\
\hline HY6464 & $M A T a(N A T) p A D H 1-t c 3-3 H A-R R M 3$ & This study \\
\hline HY6466 & MATa (NAT)pADH1-tc3-3HA-RRM3 ulp2::HIS3MX6 & This study \\
\hline HY6481 & $\begin{array}{c}\text { MATa/MATalpha ULP2/ulp2::HIS3MX6 ESC2/esc2::KanMX4 } \\
\text { SIZ2/siz2::HphNT1 }\end{array}$ & This study \\
\hline HY6475 & $\begin{array}{c}\text { MATa/MATalpha ULP2/ulp2::HIS3MX6 SGS1/sgs1::NatMX4 } \\
\text { SIZ2/siz2::HphNT1 }\end{array}$ & This study \\
\hline HY6876 & MATa rrm3::HIS3MX6 ulp2::KANMX4 ulp1-C376(URA3) & This study \\
\hline HY6874 & MATa rrm3::HIS3MX6 ulp1-C376(URA3) & This study \\
\hline HY6830 & MATa ulp2::KANMX4 ulp1-C376(URA3) & This study \\
\hline HY6771 & MATa rrm $3:: H I S 3 M X 6$ slx $5:: N a t M X 4$ & This study \\
\hline HY6772 & MATa rrm 3::HIS3MX6 ulp2::KANMX4 slx5::NatMX4 & This study \\
\hline HY6302 & MATalpha rrm $3: \because H I S 3 M X 6$ ulp $2:: K A N M X 4$ & This study \\
\hline FY1765 & MATalpha rrm $3: \because H I S 3 M X 6$ & $\begin{array}{l}\text { Lab } \\
\text { collection }\end{array}$ \\
\hline HY6862 & MATa ulp2::KANMX4 slx5::NatMX4 & This study \\
\hline HY 10382 & MATalpha SCC1-6HA::HphNT1 ULP2-9PK::HIS3MX6 & This study \\
\hline HY10485 & MATalpha SCC1-6HA::HphNT1 ULP2-9PK::HIS3MX6 wpl1::HIS3MX6 & This study \\
\hline HY10562 & $\begin{array}{c}\text { MATalpha SCC1-6HA::HphNT1 ULP2-9PK::HIS3MX6 elg } 1: \text { KanMX4 } \\
\text { wpl1::HIS3MX6 }\end{array}$ & This study \\
\hline HY10720 & MATa SCC1-6HA::HphNT1 elg1::His3MX6 wpl1::HIS3MX6 pds5::NatMX4 & This study \\
\hline
\end{tabular}

All strains are isogenic to W303 background, except for HY10570, HY10567,

HY10568, HY10569, HY10600, HY10601, HY10602, HY10599, HY10565,

HY10564, HY10566, which are derived from VG2029-7B (14), and Y2HGold (Takara).

\section{Yeast Techniques}

Yeast cultures were inoculated from overnight cultures, grown using standard growth conditions and media (15). All cultures were grown in YPD media containing glucose 
$(2 \%)$ as carbon source at $28^{\circ} \mathrm{C}$ unless otherwise indicated. For the transcriptional shutoff of genes expressed under the control of GAL promoter, cells were grown in YP Gal media containing galactose (2\%), washed once with 1 X PBS and shifted to YPD media or plated on YPD plates. For drug sensitivity assays, cells from overnight cultures were counted and diluted before being spotted on YPD plates containing the indicated concentrations of drugs and incubated at $28^{\circ} \mathrm{C}$ for $2-3$ days. For $\mathrm{Y} 2 \mathrm{H}$ analysis catalytically-dead ulp2-S624S mutant and different truncations of PDS5 were cloned into pGAD-C1 or pGBD-C1 vectors and cotransformed into Y2HGold yeast strain. Standard cloning and site-directed mutagenesis techniques were used. Maps and primer DNA sequences are available upon request.

\section{TCA Protein Precipitation}

To preserve the post-translational modifications, yeast cells were lysed under denaturing conditions. For preparation of denatured protein extracts, yeast cultures grown to an $\mathrm{OD}_{600}=0.7-1$ were pelleted by centrifugation $\left(4000 \mathrm{rpm}, 4 \mathrm{~min}, 4^{\circ} \mathrm{C}\right)$ and immediately frozen in liquid nitrogen. After thawing on ice, the pellets were lysed by addition of denaturing lysis buffer $(1.85 \mathrm{M} \mathrm{NaOH}, 7.5 \% \beta$-mercaptoethanol) for $15 \mathrm{~min}$ on ice. For the cell pellet of an $\mathrm{OD}_{600}=1$ typically $150 \mu$ of lysis buffer was used. To precipitate the proteins, the lysate was subsequently mixed with an equal volume (150 $\mu \mathrm{l}$ in case of $\left.\mathrm{OD}_{600}=1\right)$ of $55 \%(\mathrm{w} / \mathrm{v})$ trichloroacetic acid (TCA) and further incubated on ice for $15 \mathrm{~min}$. The precipitated material was recovered by two sequential centrifugation steps $\left(13000 \mathrm{rpm}, 4^{\circ} \mathrm{C}, 15 \mathrm{~min}\right)$. Pelleted denatured proteins were then either directly resuspended in HU sample buffer (8 M urea, 5\% SDS, $1 \mathrm{mM}$ EDTA, 1,5\% DTT, $1 \%$ bromophenol blue; $50 \mu \mathrm{l}$ per $\mathrm{OD}_{600}=1$ ), boiled for $10 \mathrm{~min}$ and stored at $-20^{\circ} \mathrm{C}$, or used for downstream processing, e.g., Ni-NTA pull-downs of His-tagged 
SUMO conjugates.

\section{Ni-NTA Pull-down of His SUMO Conjugates}

For isolation of in vivo SUMOylated substrates from yeast cells expressing Nterminally His-tagged Smt3 ( ${ }^{\text {His }}$ SUMO), denatured protein extracts were prepared and Ni-NTA chromatography was carried out as described previously $(2,16)$. In general, $200 \mathrm{OD}_{600}=1$ of logarithmically growing cells were harvested by centrifugation (4000 $\mathrm{rpm}, 4 \mathrm{~min}, 4^{\circ} \mathrm{C}$ ), washed with pre-chilled water, transferred to $50 \mathrm{ml}$ falcon tube and lysed with $6 \mathrm{ml}$ of $1.85 \mathrm{M} \mathrm{NaOH} / 7.5 \% \beta$-mercaptoethanol for $15 \mathrm{~min}$ on ice. The proteins were precipitated by adding $6 \mathrm{ml}$ of 55\% TCA and another $15 \mathrm{~min}$ incubation on ice (TCA-precipitation, described above). Next, the precipitate was pelleted by centrifugation $\left(3500 \mathrm{rpm}, 15 \mathrm{~min}, 4^{\circ} \mathrm{C}\right)$, washed twice with water and finally resuspended in buffer A (6 $\mathrm{M}$ guanidine hydrochloride, $100 \mathrm{mM} \mathrm{NaH}_{2} \mathrm{PO}_{4}, 10 \mathrm{mM}$ Tris-HCl, $\mathrm{pH}$ 8.0, $20 \mathrm{mM}$ imidazole) containing 0.05\% Tween-20. After incubation for 1 hour on a roller at room temperature with subsequent removal of insoluble aggregates by centrifugation $\left(23000 \mathrm{~g}, 20 \mathrm{~min}, 4^{\circ} \mathrm{C}\right)$, the protein solution was incubated overnight at $4{ }^{\circ} \mathrm{C}$ with $50 \mu \mathrm{l}$ of Ni-NTA agarose beads in the presence of $20 \mathrm{mM}$ imidazole. After incubation, the beads were washed three times with buffer A containing $0.05 \%$ Tween20 and five times with buffer $\mathrm{B}\left(8 \mathrm{M}\right.$ urea, $100 \mathrm{mM} \mathrm{NaH}_{2} \mathrm{PO}_{4}, 10 \mathrm{mM}$ Tris- $\mathrm{HCl}, \mathrm{pH}$ 6.3 ) with $0.05 \%$ Tween-20. His SUMO conjugates bound to the beads were finally eluted by incubation with $50 \mu \mathrm{l}$ of $\mathrm{HU}$ sample buffer for $10 \mathrm{~min}$ at $65^{\circ} \mathrm{C}$. Proteins were resolved on precast Bolt 4\%-12\% Bis-Tris Plus gradient gels, and analyzed by standard Western blotting techniques using antibodies listed below.

\section{Antibodies}


Mouse monoclonal anti-Viral V5-TAG antibody (1:5000; clone SV5-Pk1) was purchased from Bio-Rad/AbD Serotec. Mouse monoclonal anti-Pgk1 antibody (1:2000; clone 22C5D8) was obtained from Thermo Fisher Scientific. Mouse monoclonal antiHA antibody (1:2000; clone F-7) and rabbit polyclonal anti-Smt3 (1:2000; clone y-84) antibody were from Santa Cruz Biotechnology, as well as normal mouse IgG. Mouse monoclonal anti c-MYC antibody (1:2000; clone 9E10) was produced in house. Mouse monoclonal anti-acetyl-Smc3 antibody (17) (1:2000) was a kind gift from Katsuhiko Shirahige. Anti-rabbit IgG and anti-mouse IgG, HRP-linked antibodies (1:5000) were purchased from Cell Signaling Technology.

\section{Immunoprecipitation}

For the immunoprecipitation (IP) and binding studies involving co-IP, native yeast extracts were prepared by cell disruption using grinding in liquid nitrogen. To avoid protein degradation and loss of PTMs, lysis buffer (150 mM NaCl, 10\% glycerol, $1 \%$ NP-40, $50 \mathrm{mM}$ Tris $\mathrm{HCl}, \mathrm{pH}$ 8.0) was supplemented with inhibitors: EDTA-free complete cocktail, 20mM N-ethylmaleimide, $1 \mathrm{mM}$ phenylmethanesulfonyl fluoride (PMSF), $25 \mathrm{mM}$ iodoacetamide, and phosphatase inhibitor cocktails 2 and 3 (SigmaAldrich). For IPs, anti-PK and anti-MYC antibodies, together with recombinant protein G Sepharose 4B beads were used. IPs were performed overnight with head-over-tail rotation at $4{ }^{\circ} \mathrm{C}$ and were followed by stringent washing steps to remove non-specific background binding to the beads.

\section{ChIP-qPCR}


Chromatin immunoprecipitation (ChIP) was carried out as previously described (6). Briefly, cells were collected at the indicated experimental conditions and crosslinked with $1 \%$ formaldehyde for $30 \mathrm{~min}$. Cells were washed twice with ice-cold $1 \mathrm{X}$ TBS, suspended in lysis buffer supplemented with $1 \mathrm{mM}$ PMSF, $20 \mathrm{mM} \mathrm{NEM}$, and 1X EDTA-free complete cocktail, and lysed using FastPrep-24 (MP Biomedicals). Chromatin was sheared to a size of $300-500$ bp by sonication. IP reactions with antiHA antibodies and Dynabeads protein $\mathrm{G}$ were allowed to proceed overnight at $4{ }^{\circ} \mathrm{C}$. After washing and eluting the ChIP fractions from beads, crosslinks were reversed at $65^{\circ} \mathrm{C}$ overnight for both Input and IP. After proteinase K treatment, DNA was extracted twice by phenol/chlorophorm/isoamyl alcohol (25:24:1, v/v). Following precipitation with ethanol and Ribonuclease A (RNase A) treatment, DNA was purified using QIAquick PCR purification kit. Real-time PCR was performed using QuantiFast SYBR Green PCR kit according to the manufacturer's instructions and each reaction was performed in triplicates using a Roche LightCycler 96 system. The results were analyzed with absolute quantification $/ 2^{\text {nd }}$ derivative maximum and the $2(-\Delta \mathrm{C}(\mathrm{t}))$ method. Each ChIP experiment was repeated at least three times. Statistical analysis was performed using Student's unpaired $t$-test. The error bars represent standard error of mean (SEM).

\section{Mass Spectrometry}

For the detection of degradation-prone SUMO conjugates decreased in abundance in ulp2 2 cim3-1 mutant cells specifically in a SUMO-chain-dependent manner (Figure 1), SILAC-based mass spectrometry protocol (18) was used. Yeast ulp2 $\Delta$ cim3-1 mutant cells deficient in biosynthesis of lysine and arginine (lys $1 \Delta$ and $\arg 4 \Delta)$ expressing either wild-type His-tagged SUMO (HisSUMO) or its lysine-less variant (KRall) that 
cannot form lysine-linked polySUMO chains were grown for at least ten divisions in synthetic complete media supplemented either with unlabeled (Lys0 and Arg0; light) or heavy isotope-labeled amino acids (Lys8 and Arg10; heavy) from Cambridge Isotope Laboratories. Exponentially dividing ${ }^{H i s}$ SUMO ulp $2 \Delta$ cim3-1 cells grown in heavy media were harvested, combined with equal amount of KRall ulp $2 \Delta \operatorname{cim} 3-1$ cells grown in light media, and SUMO conjugates were isolated by using denaturing Ni-NTA pulldown. Proteins isolated following denaturing Ni-NTA pull-downs of His SUMO conjugates were separated on $4-12 \%$ Bis-Tris gel. The whole lane was excised in slices and proteins were digested with trypsin. Extracted peptides were analyzed by LCMS/MS using the Q Exactive HF mass spectrometer and identified using MaxQuant (19) software.

\section{RESULTS}

\section{SUMO chains target SMC complexes and promote their turnover}

We have used quantitative proteomics to identify SUMO substrates whose turnover is promoted by SUMO chains in the absence of the SUMO protease Ulp2 that possesses SUMO-chain-editing activity in yeast cells (20). To these ends, we used a SILAC-based mass spectrometry approach (18) and compared by denaturing Ni-NTA pull-down (Ni PD $(2,16))$ the levels of SUMO conjugates in $u l p 2 \Delta$ cim3-1 mutants expressing either endogenous yeast SUMO (SMT3) N-terminally tagged with a 7xHis-tag $\left({ }^{\mathrm{His}} \mathrm{SUMO}\right)$ or a lysine-less SUMO variant (KRall) that cannot form lysine-linked polySUMO chains (Figure 1, left). We used the temperature sensitive proteasome defective cim3-1 mutant cells at the permissive temperature of $30^{\circ} \mathrm{C}$ to allow cell cycle progression and accumulation of degradation-prone substrates, thus facilitating their identification by mass spectrometry. The SILAC screen quantified 726 potential SUMO conjugates 
(Figure 1, right); the abundance of most of them did not change significantly, while SUMO conjugates (Smt3) pulled-down from the KRall mutant were more abundant in general. Notably, among the SUMO substrates strongly enriched in the sample derived from chainless SUMO ulp2 $\Delta$ cim3-1 cells, were subunits of all three SMC complexes. SUMOylated cohesin subunits Smc1, Smc3, Scc1, Scc3, and Pds5 were enriched the most when SUMO-chain growth was prevented by the KRall mutation, whereas SUMOylated condensin subunits Smc4, Brn1, and the Smc5/6 complex subunits Smc5, Smc6, Nse4 accumulated to a lesser extent.

Cohesin shows strong ties to the SUMO system (9-11,21-23). Its regulatory subunit Pds5 is a known SUMO target ((14) and Supplementary Figure S1A) and is one of the first identified substrates of Ulp2 (14). These findings, together with the results of our SILAC screen suggesting that most of cohesin subunits are subjected to SUMOchain-mediated turnover, prompted us to focus on studying the regulation of the cohesin complex by SUMO chains and Ulp2.

We hypothesized that one of the roles of the essential regulatory cohesin subunit Pds5 is to recruit Ulp2 to protect cohesin against unscheduled SUMO-chain-mediated turnover. Indeed, we could confirm the interaction of Ulp2 with Pds5 using both coimmunoprecipitation (co-IP) and yeast two-hybrid (Y2H) studies (Supplementary Figure S1B-D). For the co-IP, we C-terminally tagged endogenous Pds5 and Ulp2 with 13Myc and 9PK tags, respectively. IPs with the anti-PK (Supplementary Figure S1B) and anti-Myc (Supplementary Figure S1C) antibodies revealed that Ulp2 interacts with Pds5 and there is preference towards upshifted, potentially SUMO-modified forms of proteins. These slower-migrating species of Pds5 and Ulp2 were coimmunoprecipitated with specific antibodies, but not mouse IgG (Supplementary Figure S1B-C). For the Y2H studies, we used Gal4 DNA-binding domain (BD) fusions 
of various Pds5 truncations and the Gal4 activation domain (AD) fusion of catalytically dead Ulp2 (Ulp2-C624S; Ulp2 $\mathrm{CD}$ ), which we expected to interact stronger with potential substrates based on previous work on Ulp1 $1_{\mathrm{CD}}$ that behaved like a SUMO substrate trap (24) (Supplementary Figure S1D-F). We observed weak Y2H interaction of Ulp2 with both N-terminal (aa 1-701) and C-terminal (aa 702-1277) fragments of Pds5 (Supplementary Figure S1D). However, analysis of further N-terminal Pds5 truncations revealed auto-activation of the HIS3 reporter gene by Pds5 N-terminus (aa 1-250, Supplementary Figure S1E), suggesting that binding of Ulp2 to Pds5 may be mediated by its $\mathrm{C}$-terminus. Indeed, using $\mathrm{C}$-terminal truncations of Pds5 we found that the C-terminal fragment of Pds5 (aa 1078-1277) is required for the interaction with Ulp2 in the Y2H system (Supplementary Figure S1F). Altogether, these results potentially support the notion that Pds5 recruits Ulp2 to prevent SUMO chain-mediated turnover of cohesin.

\section{The essential role of Pds5 relates to curbing down SUMO chains}

Cohesin plays critical roles in numerous cellular pathways $(8,25-27)$, including sister chromatid cohesion and chromosome condensation, for both of which PDS5 is required $(28,29)$. Interestingly, sister chromatid cohesion defects and lethality of the temperature sensitive pds5-1 mutant are suppressed by deletion of ELG1 (30), which acts in the context of the Elg1-Rfc2-5 complex as principal unloader of chromatin-bound proliferating cell nuclear antigen (PCNA) (31). Chromosome condensation defects of pds5-1 mutant, but not lethality, are in turn suppressed by deletion of RAD61 (WPL1) (30), which encodes the cohesin release factor WAPL that interacts with Pds5 and destabilizes cohesin's binding to DNA (32). 
To study the potential role of Pds5 in counteracting SUMO chains via Ulp2 recruitment to cohesin, we decided to examine the effect of elgl $1 \Delta$ and $w p l 1 \Delta$ mutations on the viability of PDS5 null cells when SUMO chain formation is prevented. First, we

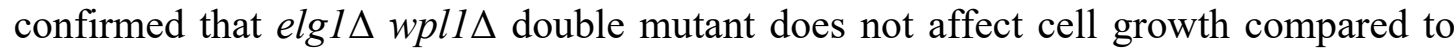
single mutants and WT cells (Figure 2A and (33)). Then, we generated ELG1/elg1A WPL1/wpl1 $\triangle$ PDS5/pds5 smt3-KRall/smt3-KRall diploid strain expressing chainless SUMO variant smt3-KRall as the only source of SUMO. The analysis of resulting haploids after sporulation and tetrad dissection of this strain should reveal if elgl$\Delta$ wpll $\Delta$ smt3-KRall triple mutant bypasses the essential role(s) of PDS5. Strikingly, we found that not only $p d s 5 \Delta$ elg $1 \Delta$ wpll $s m t 3-K R a l l$ quadruple mutant was viable, but that expression of smt3-KRall alone bypassed the requirement of PDS5 for viability (Figure 2B). We next analyzed if $\operatorname{elg} 1 \Delta \operatorname{wpl} 1 \Delta p d s 5 \Delta$ triple mutant is viable following

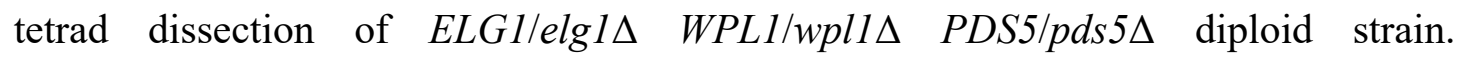
Interestingly, also the elg $1 \Delta$ wpll $\Delta$ double mutant suppressed the lethality of $p d s 5 \Delta$ cells (Figure 2C). Thus, we uncovered that the essential role of PDS5 in budding yeast

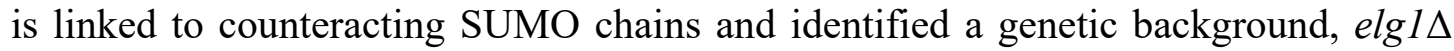
$w p l 1 \Delta$, in which this essential function is bypassed.

Next, we checked if expression of the chainless SUMO variant smt3-KRall, elg $1 \Delta$ wpll $1 \Delta$ double mutant, or their combination, is able to suppress the loss of another cohesin HAWK protein Scc3, which is required for cohesin binding to DNA (34), but not for its loading to chromatin mediated by $\operatorname{Scc} 2 / \operatorname{Scc} 4$ complex (35). None of the mutations provided viability to $\operatorname{scc} 3 \Delta$ haploids upon tetrad dissection of $E L G 1 / \operatorname{elg} 1 \Delta$

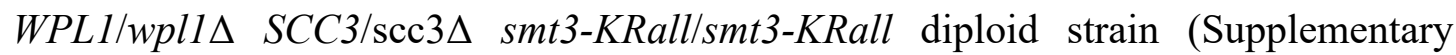
Figure S2A). The lethality of cells upon deletion of ECO1, the acetyltransferase required for Smc3 lysine K112, K113 acetylation and sister chromatid cohesion 
establishment $(36,37)$, is also not suppressed by expressing smt3-KRall and is only bypassed by wpl1 $($ Supplementary Figure S2B). Thus, while PDS5 overexpression partially suppresses the temperature sensitivity of certain ecol thermosensitive mutants (38), smt3-KRall that bypasses the essential role of PDS5 does not restore viability of cells lacking Eco1 (Supplementary Figure S2B), suggesting that other functions of $P D S 5$ are required for this suppression.

To determine the consequences of PDS5 loss for the chromatin-bound cohesin levels, we assessed Eco1-mediated Smc3 lysine K112, K113 acetylation in the identified PDS5 null bypass conditions. Ecol acetyltransferase targets cohesin loaded onto DNA (39), thus making Smc3 acetylation a good indicator of the functionallyengaged cohesin amounts operating in the cell. To this end, we utilized a monoclonal antibody specific for the Eco1-mediated Smc3 lysine K112, K113 acetylation (17) and found that it was largely reduced, but not abolished, in all $p d s 5 \Delta$ mutants (Figure 2D and Supplementary Figure S2C). Thus, cells lacking Pds5 have reduced levels of chromatin-bound cohesin available to fulfil its functions, which is in agreement with findings in pds5-ts cells $(11,29)$. We further found that while limited amounts of chromatin-bound acetylated cohesin in $p d s 5 \Delta \operatorname{elg} 1 \Delta$ wpll $\Delta$ mutants are sufficient to support viability, the mutant cells are sensitive to low and high temperatures, replication stress induced by hydroxyurea (HU), exposures to the DNA-alkylating agent methyl methanesulfonate (MMS), topoisomerase poison camptothecin (CPT) and microtubuledepolymerizing drug benomyl (Figure 2E).

Loss of WPL1 was previously reported to cause an increase in pericentromeric

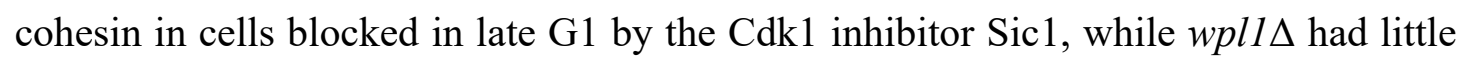
or no effect on the extent of Scc1 association with chromosome arms (40). To provide insights on how elgl $1 \Delta p l 1 \Delta$ mutant suppresses the lethality of $p d s 5 \Delta$ cells, we next 
analyzed the cohesin levels on chromatin at the pericentromeric region of CEN10, TER1004, and the centromere-distal region on chromosome 3, ARS305, by performing chromatin immunoprecipitation (ChIP) of C-terminally 6HA-tagged Scc1 from

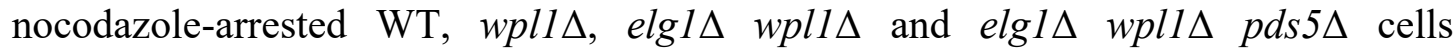
(Supplementary Figure S2D-E). Interestingly, we observed statistically significant increase of Scc1 association in $\operatorname{wpll\Delta }$ and $\operatorname{elg} 1 \Delta$ wpll $\Delta$ mutants compared to WT specifically at the pericentromeric region (Supplementary Figure S2D), but not at the chromosome arm (Supplementary Figure S2E). Depletion of Pds5 using an auxininducible degron system in G1-arrested cells was shown previously to increase genome-wide Scc1 chromatin association two-fold (40). The authors suggested that Pds5 negatively regulates Scc2-mediated cohesin loading throughout the genome. In line with their findings, we observed a two-fold increase in Scc1 chromatin levels at

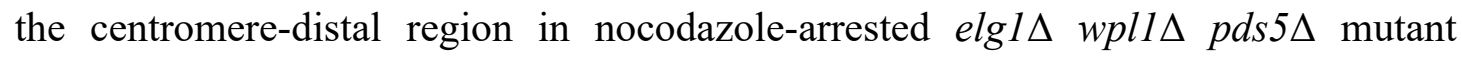
compared to WT and elgl $1 \Delta p l 1 \Delta$ cells (Supplementary Figure S2E). Scc1 chromatin levels at the pericentromeric region in the $\operatorname{elg} 1 \Delta$ wpll $1 \Delta$ pds $5 \Delta$ were also increased at least two-fold compared to WT, however no further increase was observed compared to elg $1 \Delta$ wpll $\Delta$ double mutant (Supplementary Figure S2D). Elevated Scc1 chromatin loading mediated by Scc 2 in the absence of Pds5 as assessed by ChIP does not however provide information regarding the fate of the loaded cohesin complexes, which may be targeted by subsequent SUMO-chain-mediated turnover in PDS5 null cells causing a major reduction of Ecol-mediated Smc3 acetylation levels (Figure 2D and Supplementary Figure S2C).

Ulp2 protease is essential in pds5 $\operatorname{elg} 1 \Delta$ wpl1 $\Delta$ cells and its loss is bypassed by spontaneous suppressor of $u l p 2 \Delta$ 


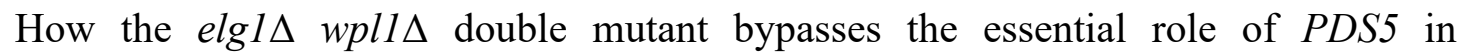
counteracting SUMO chains is unclear, but we speculated that the viability of $p d s 5 \Delta$ elg $1 \Delta$ wpl $1 \Delta$ triple mutant may depend on the presence of the Ulp2 protease as the only source of SUMO-chain-editing activity in yeast cells. To test our hypothesis, we replaced the endogenous promoter of ULP2 with the galactose-inducible $G A L$ promoter, whose transcription can be inhibited by shifting cells from galactosecontaining YP Gal to glucose-containing YPD media. Importantly, upon transcriptional

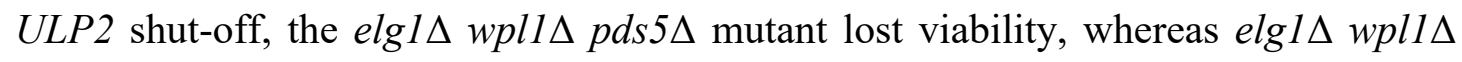

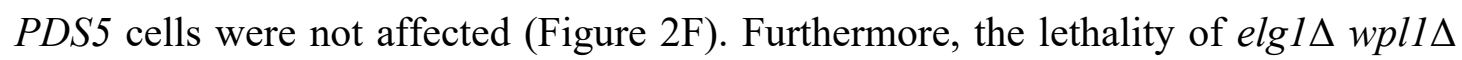
pds5 mutant following Ulp2 depletion could be suppressed by a spontaneous suppressor of $u l p 2 \Delta$ phenotypes that we identified and denoted as $u l p 1-C 376$ (Figure 2G), described below (Supplementary Figure S3).

Cells lacking Ulp2 exhibit a pleiotropic phenotype that includes temperaturesensitivity and increased sensitivity to hydroxyurea (41). We performed a screen for spontaneous suppressors of $u l p 2 \Delta$-associated HU sensitivity and isolated 5 suppressors that also alleviated the temperature sensitivity (Supplementary Figure S3A). Backcrossing isolated suppressors to the $u l p 2 \Delta$ mutant of the opposite mating type, revealed $2^{+}: 2^{-}$segregation of HU sensitivity (Supplementary Figure S3B), which points to a single mutated gene locus responsible for the suppression. Whole genome sequencing (WGS) of the isolated suppressors led to the identification of a single point mutation on chromosome 16 at the $Y P L 020 C$ (ULP1) gene in all five suppressors, but not in WT or ulp $2 \Delta$ cells (Supplementary Figure S3C). Ulp1 is the second yeast SUMO protease besides Ulp2. Differently from Ulp2, it does not have preference for SUMO chains, is essential for the maturation of conjugatable SUMO from its precursor polypeptide, and is anchored to nuclear pore complexes (NPC) via its N-terminus (41- 
45). Re-sequencing of the ULP1 locus validated the WGS results and confirmed a single insertion c.741_742insA denoted as ulp1-sup (Supplementary Figure S3D). This insertion results in a frame shift predicted to generate a C-terminally truncated Ulp1 variant p.Val248Serfs*7 lacking the protease domain (Supplementary Figure S3E), a notion not consistent with the essential nature of Ulp1 and its protease domain (Supplementary Figure S3F-G) and different from the isolated ulp1-sup (Supplementary Figure S3H-I). Careful analysis of the ULPI sequence revealed an alternative transcription/translation start site upstream of the frame shift mutation that might be used in ulp1-sup causing the expression of an N-terminally truncated Ulp1 variant (Supplementary Figure S3I) with a new N-terminal extension of 7 amino acids upstream of Lys246 (Supplementary Figure S3J). To confirm that ulp1-sup is indeed generating an N-terminally truncated Ulp1 variant able to suppress $u l p 2 \Delta$ phenotypes and provides viability as the only source of SUMO protease activity in the cell, we constructed the $u l p 1-C 376$ mutant (Supplementary Figure S3K) by replacing $u l p 1 \Delta$ in

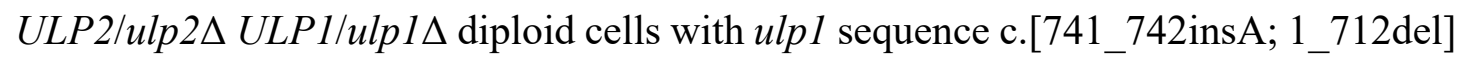
that lacks the N-terminal NPC-targeting region (aa 1-245; first 712 nucleotides of ULP1 ORF deleted). Tetrad dissection of the resulting ULP2/ulp2 $\triangle$ ULP1/ulp1-C376 diploids (Supplementary Figure S3L) revealed that ulp1-C376 mutant suppressed the HU and temperature sensitivity of $u l p 2 \Delta$ cells similar to $u l p 1$-sup (Supplementary Figure S3M). Because Ulp1-C376 is expressed at very low levels from the alternative start site compared to wild-type Ulp1 (Supplementary Figure S3I), it does not lead to cell death due to excessive deSUMOylation observed upon expression of N-terminal Ulp1 truncations at higher levels $(43,45)$.

Taken together, the identified spontaneous suppressor of $u l p 2 \Delta$ phenotypes ulp1-C376 is the N-terminal truncation of Ulp1 that is no longer tethered to the NPC 
and is able to deSUMOylate Ulp2 substrates in the nucleoplasm providing viability to otherwise lethal elg $1 \Delta$ wpl1 1 pds5 4 ulp2 quadruple mutant (Figure 2G). Thus, the viability of $p d s 5 \Delta$ elg1 $w p l 1 \Delta$ cells depends on Ulp2 ability to counteract SUMO chains and can be bypassed by inducing limited deSUMOylation with the ulp1-C376 suppressor of $u l p 2$ phenotypes.

\section{Loss of Pds5 triggers SUMOylation of cohesin's kleisin Scc1 that is counteracted}

\section{by Ulp2}

We showed that SUMOylated cohesin subunits are targeted by SUMO chains for turnover in the absence of Ulp2 (Figure 1); that the cohesin's regulatory subunit Pds5 is no longer essential when SUMO chains cannot form (Figure 2B); and that $p d s 5 \Delta$ elg $1 \Delta$ wpll $\Delta$ cells rely on Ulp2 activity or may survive when N-terminal Ulp1 truncation not tethered to the NPC can perform Ulp2 functions (Figure 2F-G). Next, we aimed to address whether Ulp2 together with Pds5 indeed protects SUMOylated cohesin from SUMO-chain-mediated turnover.

In line with previous observations in a pds5-1 mutant (11), we find that the lethality due to transcriptional PDS5 shut-off is suppressed by slx $5 \Delta$ and expression of chainless SUMO smt3-KRall variant (Supplementary Figure S4A). Importantly, conditional depletion of Pds 5 expressed under the $G A L$ promoter allowed us to follow the induction of cohesin SUMOylation and turnover (Figure 3A) by monitoring SUMO modification of cohesin's kleisin Scc1 and its Slx5/8-targeted proteasome-mediated degradation, previously shown to be enhanced in $p d s 5-1$ (11). Scc1 monoSUMOylation is detected at low levels prior to PDS5 shut-off (induced by shift to glucose-containing

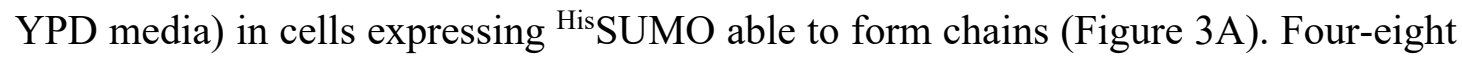
hours after the shift to YPD, Pds5 levels drop while monoSUMOylated Scc1 species 
massively accumulate and di/triSUMO-modified Scc1 can be detected. Pds5 depletion is accompanied by reduction in Smc3 lysine K112, K113 acetylation levels and degradation of the unmodified Scc1 (Figure 3A, Input), which is hardly detected 20 hours after PDS5 shut-off and leads to loss of its SUMOylation. Importantly, expression of the chainless SUMO variant KRall results in accumulation of monoSUMOylated Scc1 species even prior to Pds5 depletion (Figure 3B, compare time point 0 in KRall versus His SUMO background), suggesting that when SUMO-chain formation is prevented, monoSUMOylated Scc1 species become more stable. Moreover, deletion of ULP2 in the KRall background leads to further accumulation of monoSUMOylated Sccl species now readily detectable also in the Inputs and accompanied by increase in di/triSUMO-modified Scc1 species (Figure 3B, KRall ulp2 $2 \Delta$ background). The latter suggests that Ulp2 is able to either cleave off SUMO of mono/multiSUMOylated Scc1 or that physical interaction of Ulp2 with cohesin prevents Scc1 SUMOylation, similar to what has been proposed for Pds5 (11). Because expression of the catalytically inactive ulp2-C624S mutant results in similar Scc1 multiSUMOylation levels as observed in $u l p 2 \Delta$ mutant (Supplementary Figure S4B), we conclude that Ulp2 is able to cleave off SUMO of mono/multiSUMOylated Scc1. Notably, when SUMO chains are able to form ( ${ }^{\mathrm{His} S U M O}$ expressed), the contribution of Ulp2 to the protection of monoSUMOylated Scc1 species is hardly visible in Ni PD and is manifested by the accumulation of diSUMOylated Scc1 species in ulp2 $\Delta$ cells (Supplementary Figure $\mathrm{S} 4 \mathrm{C}$ ), in contrast to the KRall background (Figure 3B, compare time point 0 in KRall versus KRall ulp2A) where mono/multiSUMOylated Scc1 species are no longer converted to the degradation-prone polySUMOylated species and accumulate. Taken together, these data reveal a role for Ulp2 in guarding mono/multiSUMOylated Scc1 
species of cohesin against SUMO-chain-mediated proteasomal turnover that is induced upon Pds5 loss.

\section{Fusion of Ulp2 to Scc1 supports viability in the absence of Pds5 and protects cohesin from SUMO-chain-mediated turnover}

To unambiguously probe whether Ulp2 guards cohesin against SUMO-chain-targeted proteasomal degradation, we next asked if fusion of Ulp2 to the cohesin's kleisin Scc1 can suppress the lethality of cells lacking Pds5. Previously, fusion of the catalytically active Ulp1 domain to Scc1 (Scc1-UD) was shown to promote loss of cohesin's SUMO modifications, including monoSUMOylation of Smc3 and Scc1-UD itself (9). Scc1UD failed to complement the temperature sensitive phenotype of scc1-73 cells and showed sister chromatid cohesion defects, emphasizing the importance of cohesin SUMOylation for this process. Differently from Scc1-UD that abolishes cohesin SUMOylation, Ulp2 fusion to Scc1 should specifically antagonize its polySUMOylation in the absence of Pds5 leaving mono/multiSUMOylation intact and possibly stabilizing its kleisin.

To this end, we C-terminally fused the Ulp2 fragment (aa 1-734) to the endogenous Scc1 without any linker, providing Scc1-Ulp2 fusion scc1-ulp2 ${ }_{734}-6 H A$ as the only source of cohesin's kleisin in the cell (Figure 4A). The fused Ulp2 fragment contains N-terminal SIMs required for recognition of SUMO chains (6) and a protease domain, but is lacking its C-terminal 300 amino acids where sequences that mediate its localization to the nucleolus and the inner kinetochore reside $(46,47)$. Additionally, we established catalytically dead $s c c 1-u l p 2{ }_{734-C 624 S-6 H A}$ fusion with Ulp2 active site cysteine mutated (Figure 4A) and scc1-ulp2 ${ }_{734}$-sim- $6 H A$ fusion having Ulp2 N-terminal SIMs required for robust SUMO-chain-binding mutated (Supplementary Figure S5A). 
First, we validated that expression of these fusions as the only source of cohesin's kleisin in WT cells supports viability and that different fusions are expressed at similar levels (Supplementary Figure S5B). Next, we confirmed that expression of the Ulp2 catalytically dead scc1-ulp2 ${ }_{734}-C 624 S-6 H A$ fusion in the $p d s 5 \Delta$ elg $1 \Delta$ wpll $\Delta$ background is also tolerated (Supplementary Figure S5C).

Importantly, catalytically active $s c c 1-u l p 2{ }_{734}-6 H A$, but not catalytically dead scc1-ulp2 ${ }_{734-C 624 S-6 H A}$ nor scc1-ulp2 ${ }_{734-}$-sim- $6 H A$ fusion defective in binding to SUMO chains, was able to efficiently suppress the lethality of cells upon PDS5 transcriptional shut-off (Figure 4B). Interestingly, if cells were allowed to grow longer on YPD plates, minor suppression of lethality induced by Pds5 depletion was also observed in cells expressing scc1-ulp2 ${ }_{734}-$ sim- $6 H A$ fusion (Supplementary Figure $\mathrm{S} 5 \mathrm{D})$, suggesting that when fused to Scc1, Ulp2 is able to antagonize polySUMOylation even when SUMO-chain recognition is compromised. In the same line, scc1-ulp2734$6 H A$ fusion, but not catalytically dead scc1-ulp2 ${ }_{734}-C 624 S-6 H A$ provided for viability of $p d s 5 \Delta$ elgl $1 \Delta$ wl1 $\Delta$ cells upon glucose-induced transcriptional shut-off of ULP2 (Figure 4C).

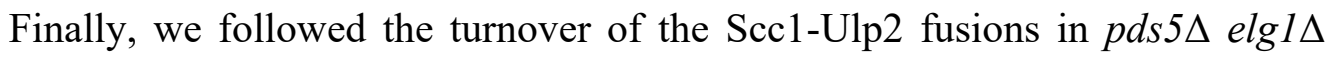
wpl1 $\triangle$ cells upon ULP2 shut-off and found that while the catalytically dead fusion is largely degraded 8 hours after Ulp2 depletion (Figure 4D), the catalytically active fusion remains stable (Figure 4E).

Moreover, we followed the SUMOylation status of these fusions upon ULP2 shut-off by performing Ni PD of His SUMO conjugates able to form SUMO chains (Figure 5A-B). Catalytically active Scc1-Ulp2 fusion remained monoSUMOylated (Figure 5A, Ni PD) and Smc3 lysine K112, K113 acetylation levels did not change significantly upon depletion of Ulp2 (Figure 5A, Input). In contrast, catalytically dead 
Scc1-Ulp2 fusion became excessively SUMOylated after four hours of Ulp2 depletion

(Figure 5B, Ni PD), monoSUMOylated species were converted to slower-migrating polySUMOylated species and finally degraded resulting in the loss of unmodified Scc1Ulp2 fusion after eight and twenty hours (Figure 5B, Input). Smc3 lysine K112, K113 acetylation levels were also largely reduced (Figure 5B), similar to the situation upon PDS5 shut-off (Figure 3A). Taken together, these experiments demonstrate that Ulp2 protects cohesin from SUMO-chain-mediated proteasomal turnover in collaboration with Pds5, and that the essential function of budding yeast Pds5 is to counteract SUMOchain targeting of cohesin, which can be bypassed either by expression of chainless SUMO variant (Figure 2B) or by the Ulp2 fusion to cohesin.

\section{Condensin is protected by Ulp2 against SUMO-chain-mediated turnover}

Condensin subunits are known SUMO substrates (22), however the functional significance of their modification is not clear. Recently, suppressor screening in fission yeast revealed that mutants of condensin's non-SMC subunits are rescued by impairing the SUMOylation pathway (48). Moreover, deleting ULP2 was synthetically lethal with the fission yeast temperature sensitive $c u t 3 /$ smc4 mutant at permissive temperatures for cut3-477, while the $u l p 2 \Delta$ mutant showed defective chromosome condensation (49).

In our SILAC-based proteomics screen, we identified condensin subunits Smc4 and Brn1 as potential substrates of SUMO-chain-targeted turnover in cells lacking Ulp2 (Figure 1). Furthermore, overexpression of $U L P 2$ was found to suppress the temperature sensitivity of the $s m c 2-6$ mutant, while $u l p 2 \Delta$ cells were defective in enriching condensin on mitotic chromatin, in particular at rDNA $(50,51)$. We reasoned that similar to cohesin, the condensin complex may be guarded by Ulp2 against SUMO-chain-mediated turnover. We first used genetic analysis to strengthen our hypothesis. While ULP2 
overexpression suppresses smc2-6(50) and smc2-8(14) alleles, we found that $u l p 2 \Delta$ has synergistic growth defect with temperature sensitive smc2-8 mutant (Figure 6A) at temperatures permissive for the single mutants, similar to fission yeast cut3/smc4 (49), suggesting that in the absence of Ulp2 the function of condensin is further compromised in $s m c 2-8$ cells. Importantly, the above-mentioned synthetic lethality of $s m c 2-8$ ulp $2 \Delta$ cells is rescued by expressing as the single source of SUMO the smt3-KRall SUMO variant that cannot form lysine-linked SUMO chains (Figure 6B).

In cohesin, the lethality caused by depletion of the HAWK protein subunit Pds5 is suppressed by the expression of smt3-KRall (Figure 2B) and is accompanied by massive SUMOylation of cohesin's kleisin Scc1 (Figure 3). However, this was not the case when we conditionally depleted the essential HAWK proteins of condensin, Ycg1 and Ycs4, in the smt3-KRall background (Supplementary Figure S6A), emphasizing that protection of condensin against SUMO chains is not their essential function. Furthermore, multiSUMOylation of condensin's kleisin Brn1 tagged C-terminally with 6HA slowly decreased following transcriptional shut-off of either YCG1 (Supplementary Figure S6B) or YCS4 (Supplementary Figure S6C), contrary to multiSUMOylation of cohesin's kleisin Scc1 upon Pds5 depletion (Figure 3A). Nevertheless, the synthetic lethality of $s m c 2-8$ ulp $2 \Delta$ cells was suppressed by expressing smt3-KRall (Figure 6B) and deleting SLX5 (Figure 6C and Supplementary Figure S6D). Taken together, these genetic studies support a model in which Ulp2 guards condensin against SUMO-chain-targeted and Slx5/8 STUbL-mediated turnover.

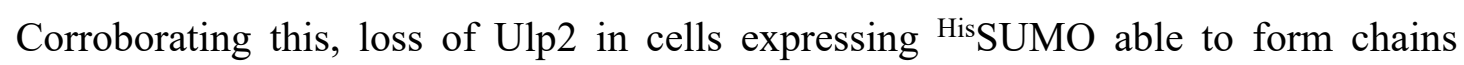
resulted in the decrease of monoSUMOylated Brn1 species compared to WT cells detected by Ni PD, whereas expression of the chainless SUMO mutant KRall not only increased the abundance of monoSUMOylated Brn1 in WT cells, but also suppressed 
the observed drop in ulp2 $\Delta$ mutant (Figure 6D). However, monoSUMOylated Brn1 species are not particularly prone to proteasomal degradation, as they are not stabilized in the temperature sensitive $\operatorname{cim} 3-1$ proteasome defective mutant (Supplementary Figure S6E) grown at permissive temperature. Overall, the results suggest that in the absence of Ulp2, SUMO chains might target the condensin complex for disassembly and release from chromatin.

\section{The Smc5/6 complex is protected by Ulp2 against SUMO-chain-mediated turnover}

We next studied if the third SMC complex Smc5/6 is similarly regulated. First, using a genetic approach, we found that $u l p 2 \Delta$ has synergistic growth defects with the temperature sensitive smc6-56 mutant (Figure 7A) at temperatures permissive for the single mutants, suggesting that in the absence of Ulp2, the function of the Smc5/6 complex is further compromised, similar to condensin (Figure 6A).

We then checked if expression of the chainless SUMO variant smt3-KRall is able to suppress either smc6-56 or loss of the essential non-SMC element proteins Nse3 and Nse5. Neither the temperature sensitivity of nse3-ts-12 and smc6-56 mutants, nor the lethality upon depletion of Nse5-AID using the auxin-inducible degron system (52) was suppressed by smt3-KRall (Supplementary Figure S7A-C). Rather, we observed synthetic sick/lethal genetic interaction between smc6-56 and smt3-KRall at temperatures permissive for the single mutants (Supplementary Figure S7C). Previously, smc6 mutations were shown to be synthetic sick/lethal with mutation of Sgs1 (53), whereas sgs $1 \Delta$ cells require SUMO-chain formation to survive and die upon expression of smt3KRall (54). Therefore, the synthetic lethality of smc6-56 smt3-KRall may be explained by the compromised function that is fulfilled by the Smc5/6 complex in collaboration 
with Sgs1 (53). The non-SMC element of the Smc5/6 complex Mms21 is a SUMO ligase that is responsible for Smc5/6 complex autoSUMOylation targeting primarily the Smc5 subunit (55). However, the Siz2 SUMO ligase also contributes to Smc5 SUMOylation (56). Because we cannot use smt3-KRall to suppress the synthetic lethality of smc6-56 ulp $2 \Delta$ double mutant, we checked if loss of Siz2 is able to do so. Previously, the temperature sensitivity of cohesin's $p d s 5-1$ mutant was suppressed by siz2s (11). We found the same to be true also for smc6-56 ulp2s (Figure 7B) and smc6-P4 $u l p 2 \Delta$ (Supplementary Figure S7D) cells. Moreover, the synthetic growth defect of smc6-P4 ulp2A double mutant is suppressed by deletion of the STUbL subunit Slx5 (Supplementary Figure S7E). In addition, $u l p 2 \Delta$ showed synthetic lethal/sick interactions with mutations in Esc2, Sgs1 and Rrm3 (Supplementary Figure S7F-I), previously found to function jointly with $\mathrm{Smc5/6}$ to facilitate resolution of recombination intermediates (Esc2, Sgs1) (57,58) and replication through difficult-to-replicate regions (Rrm3) (53).

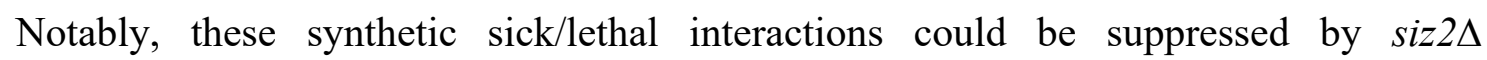
(Supplementary Figure S7J-K), ulp1-C376 (Supplementary Figure S7L) and slx54 (Supplementary Figure S7M). Taken together, these results suggest that also the Smc5/6 complex is negatively regulated by Siz2-mediated polySUMOylation, which, when not antagonized by Ulp2, is being recognized by the Slx 5/8 STUbL and targets the complex for turnover.

In line with genetics, we found that monoSUMOylated species of Nse4, the kleisin of the Smc5/6 complex, accumulate in $\operatorname{sl} 55 \Delta$ and decrease in abundance in $u l p 2 \Delta$ compared to WT cells (Figure 7C). Moreover, similar to condensin's kleisin Brn1 (Supplementary Figure S6E), monoSUMOylated Nse4 species are not particularly prone to proteasomal degradation as they are not further enriched in $u l p 2 \Delta \operatorname{cim} 3-1$ mutant compared to ulp2 $2 \Delta$ cells (Figure 7D). However, in Ni PD experiments, we find that 
monoSUMOylated Nse4 strongly accumulates in both WT and ulp2 $\Delta$ when chainless SUMO variant KRall is expressed instead of His SUMO (Figure 7E). This indicates that in the absence of Ulp2, SUMO chains might target the Smc5/6 complex for disassembly and release from chromatin, as observed for condensin.

\section{DISCUSSION}

Genetic links between the SUMO protease Ulp2 and components of both condensin (50) and cohesin (14) are known for two decades, yet the role of Ulp2 in the regulation of these SMC complexes has remained elusive. Here, we uncover that Ulp2 acts as a guardian of all three SMC complexes by protecting them from unscheduled SUMOchain-targeted turnover, thus giving them time to fulfil their functions on chromatin.

Specifically, using unbiased SILAC-based proteomic screen to identify SUMO conjugates that decrease in abundance in the absence of Ulp2 in a SUMO-chaindependent manner, we found subunits of all SMC complexes (Figure 1). Since ULP2 overexpression was initially found to suppress phenotypes of $p d s 5-1$ (14), we hypothesized that one important role of Pds5 is to recruit Ulp2 to protect cohesin from polySUMOylation. Strikingly, we showed that Pds5 not only binds Ulp2 (Supplementary Figure S1), but that the essential role of Pds5 is to counteract SUMO-chain formation, as $p d s 5 \Delta$ lethality is suppressed by expressing a lysine-less SUMO variant (KRall) that cannot form SUMO chains (Figure 2B). Moreover, we found that this essential function of Pds5 can by bypassed by combined loss of ELG1 and WPL1 (Figure 2C), individual deletions of which suppress cohesion and condensation defects of $p d s 5-1$, respectively (30). Notably, the viability of $p d s 5 \Delta$ elg $1 \Delta$ wpll $\Delta$ cells relies on Ulp2 (Figure $2 \mathrm{~F}$ ) or could be supported in its absence by expressing the $u l p 1-C 376$ spontaneous suppressor of $u l p 2 \Delta$ phenotypes (Figure $2 \mathrm{G}$ ), which gains ability to deSUMOylate Ulp2 substrates. 
We then uncovered that cohesin's kleisin Scc1 is in fact a Ulp2 substrate, and loss of Pds5 induces its polySUMOylation and subsequent degradation (Figure 3). Finally, we found that fusion of catalytically active Ulp2 to Scc1 suppresses the lethality of cells upon Pds5 loss. Furthermore, Scc1-Ulp2 fusion rescues the lethality of

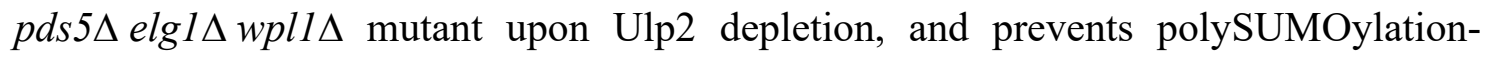
targeted degradation of cohesin's kleisin (Figures 4-5). Thus, our data reveal that Ulp2 guards functional mono/multiSUMOylated cohesin pool loaded onto DNA $(9,10)$ against unscheduled SUMO-chain-mediated turnover, providing the time window for its action until cohesin presence is no longer required, e.g. in mitosis to allow sister chromatid separation.

Interestingly, Ulp2 interacts with and is negatively regulated by the Polo-like kinase Cdc5 in mitosis, with impact on cohesion (59). CDC5 overexpression causes centromeric cohesion defects that are suppressed by additionally overexpressing ULP2. Moreover, $C D C 5$ overexpression results in Pds5 dissociation from mitotic chromosomes in pre-anaphase cells, whereas co-overexpression of ULP2 restores normal Pds5 chromosomal association (59). These findings suggest that Cdc5-mediated inactivation of Ulp2 induces SUMO-chain-targeted and S1x5/8 STUbL-mediated proteasomal degradation of the chromatin-bound mono/multiSUMOylated cohesin pool, ensuring its timely removal from chromosomes in collaboration with separase. Thus, yeast Cdc5 kinase promotes sister chromatid separation by phosphorylating Scc1 to enhance separase-mediated cleavage (60), and in parallel, by inducing SUMO-chain-mediated cohesin turnover through inactivation of Ulp2. Supporting this model, overexpression of ULP2 in separase mutant esp1-1 results in synthetic sick genetic interaction even at permissive temperatures for esp1-1 (11). 
If the essential function of Pds5 is to help counteract polySUMOylation of cohesin mediated by Ulp2, why is deletion of PDS5 lethal whereas $u l p 2 \Delta$ cells are viable? The presence of Ulp2, however, becomes essential in the $p d s 5 \Delta$ mutant, the lethality of

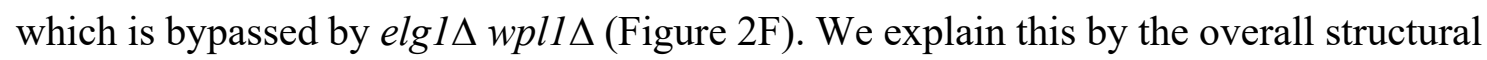
organization of the SMC complexes $(7,8)$ and of cohesin in particular $(61,62)$, and by the promiscuity of the SUMOylation enzymes (3). Specifically, the SUMO conjugating enzyme Ubc9 efficiently targets any accessible lysine able to enter its active site in vitro without additional requirements of SUMO ligases. This feature makes the highly unstructured kleisin with many exposed lysines a perfect substrate for SUMOylation, unless it is bound by structured kleisin-associated regulatory subunits. In fact, human Scc1 is SUMOylated at multiple sites and mutation of 15 lysines to arginines reduces, but does not abolish its SUMOylation (21), whereas yeast Scc1 is multiSUMOylated on at least 11 lysines (10). The deletion of PDS5 has a number of consequences for cohesin. First, Scc2-mediated cohesin DNA loading is strongly stimulated as there is no Pds5 to antagonize it (40) bringing more cohesin complexes in the vicinity of the DNA-bound SUMO ligases. Second, polySUMOylation at multiple acceptor sites on Scc1 is induced, because Pds5 is no longer available to bind Scc1, whereas Scc2 interaction with Scc1 is likely very dynamic as proposed previously (40). In the above-mentioned study, the authors fail to detect an increase in Scc2 association with the genome upon Pds5 depletion and speculate that Scc2 turnover is too rapid for efficient formaldehyde fixation. Since Scc1 is a highly unstructured protein, its exposed lysines can be readily targeted by the rather promiscuous SUMO conjugation machinery, if not shielded by Pds5. Third, loss of Pds5 abolishes recruitment of its interactors, including the SUMO protease Ulp2, to cohesin. Altogether, loss of Pds5 leads to increased Scc2-mediated DNA loading of cohesin with subsequent polySUMOylation and STUbL-mediated 
proteasomal degradation, exhausting the available pools of the unmodified Scc1. We show that SUMO-chain build-up and kleisin turnover can be prevented if Ulp2 is fused to Scc1 (Figures 4 and 5), providing viability to cells lacking Pds5.

The suppression of the $p d s 5 \Delta$ mutant lethality by elg $1 \Delta$ wpll $\Delta$, where polySUMOylation of kleisin still takes place and the presence of Ulp2 is required to antagonize it and support viability, indicates that $\operatorname{elg} 1 \Delta$ wpll $\Delta$ associated suppression is achieved by increasing the amounts of cohesin loaded onto DNA rather than by directly antagonizing polySUMOylation. Wpl1 is a cohesin release factor (32), whereas Elg1 unloads PCNA, which is important for recruiting Ecol to replication forks to promote cohesion during S phase (63). Interestingly, ECO1 overexpression suppresses pds5-1 temperature sensitivity (38), probably via Ecol-mediated Smc3 acetylation that antagonizes Wpll-mediated cohesin release $(36,37)$. The increase of cohesin DNAloading provided by elg $1 \Delta$ wpll $\Delta$ is likely restricted to specific loci in yeast, as we did not observe pronounced increase in the Eco1-mediated Smc3 lysine K112, K113

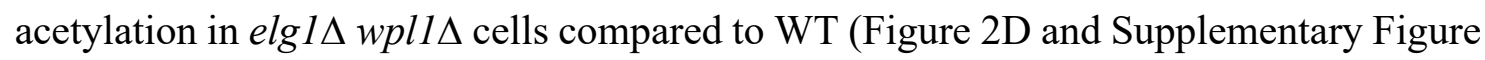
$\mathrm{S} 2 \mathrm{C})$. However, this mild increase in cohesin is sufficient to support viability of pds $5 \Delta$

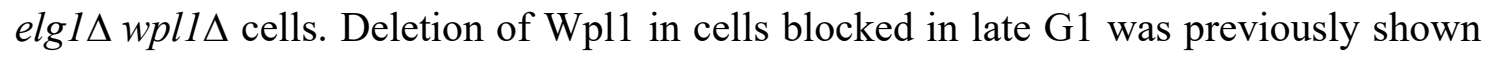
to cause a major increase specifically in peri-centric cohesin, as monitored by Scc1 chromatin binding (40), whereas conditional depletion of Pds5-AID increased Scc2mediated cohesin loading throughout the genome two-fold. Interestingly, in line with the above-mentioned findings, we observed statistically significant increase of Scc1

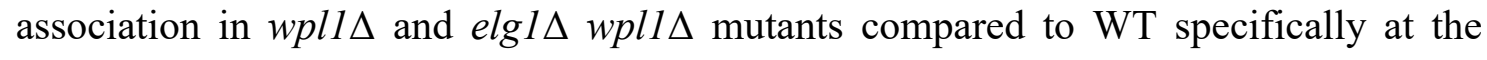
pericentromeric region, but not at the chromosome arm in nocodazole-arrested cells (Supplementary Figure S2D-E), which suggests that elg $1 \Delta$ wpll $\Delta$ might provide viability to $p d s 5 \Delta$ cells by supporting pericentromeric cohesion. Moreover, we also observed a 
two-fold increase in Scc1 loading at the centromere-distal region in nocodazole-arrested

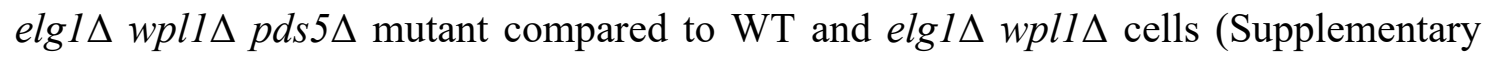
Figure S2E). Interestingly, increased topological DNA association of cohesin in nocodazole-arrested $p d s 5-101$ cells shifted to restrictive temperature compared to WT cells was reported previously (64), as monitored by the accumulation of monomeric supercoiled DNAs (CMs) in a minichromosome assay. Importantly, however, the authors observed in pds5-101 cells a marked reduction in the accumulation of DNA-DNA concatemers (CDs) that mediate chromatid cohesion by co-entrapment of sister DNAs inside cohesin rings. Thus, despite having increased Scc2-mediated cohesin DNA loading, loss of Pds5 results in decreased Smc3 acetylation levels (Figure 2D and Supplementary Figure S2C), loss of sister chromatid cohesion as monitored by minichromosome assay (64) and cell lethality. In our work, we demonstrate that the lethality of $p d s 5 \Delta$ cells is suppressed by expression of a SUMO variant unable to form chains (Figure 2B) or by fusing the SUMO protease Ulp2 that trims SUMO chains to the cohesin's kleisin Scc1 (Figure 4B-C). The fusion of catalytically active Ulp2 to Scc1 prevents its polySUMOylation and subsequent Scc1 kleisin turnover via proteasomal degradation (Figure 5), providing viability to cells lacking Pds5.

Similar to cohesin, Ulp2 guards the other two SMC complexes, condensin and the Smc5/6 complex, protecting them from SUMO-chain-targeted S1x5/8 STUbLmediated turnover, as demonstrated by genetic interaction studies and the analysis of their kleisin SUMOylation in relevant mutants of the SUMO/ubiquitin pathway. The mechanism of Ulp2 action is similar, yet it likely results in preventing the disassembly and removal of the complexes from chromatin, rather than massive proteasomal degradation. Accordingly, monoSUMOylated species are not further enriched in the proteasome defective cim3-1 mutant, but are nevertheless stabilized upon expression of 
the chainless SUMO variant KRall. Chromatin extraction of polySUMOylated and subsequently polyubiquitylated SMC complexes is likely mediated by the action of the Cdc48/p97 segregase $(65,66)$, which was previously shown to mobilize cohesin and condensin from chromatin $(67,68)$. Despite this difference, the outcome of Ulp2 loss for all SMC complexes is the same in that their turnover on chromatin is increased. Another difference is that depletion of condensin's HAWKs or the KITEs of the Smc5/6 complex is not suppressed by expressing the KRall SUMO mutant, suggesting that it is not their essential function to counteract kleisin polySUMOylation, contrary to Pds5. We note that Pds5 is likely a special case, as it is not stimulating the ATPase activity required for the DNA-loading of cohesin, in contrast to the Scc2 HAWK, with which Pds5 competes and then replaces to stabilize cohesin complex in the DNA-bound state (40).

In conclusion, here we uncovered that the SUMO protease Ulp2 acts on all three SMC complexes to protect them from unscheduled SUMO-chain-targeted turnover, giving them time to perform their essential functions on chromatin. Since Ulp2 discovery two decades ago (41), multiple phenotypes have been associated with its loss, including genome instability, sister chromatid cohesion and condensation defects. Our finding helps to explain many of them and highlights a new layer of the SMC complex regulation, through which their chromatin abundance is instructed.

\section{SUPPLEMENTARY DATA}

Supplementary Data are available at NAR online.

\section{ACKNOWLEDGEMENTS}

We thank Eurofins Genomics for the whole genome sequencing of yeast strains, mapping the sequencing reads and variant analysis, M. Foiani, S. Jentsch, and K. 
Shirahige for sharing reagents, A. Cattaneo and A. Bachi for mass spectrometry analysis, L. Fendillo for help with generating Scc1-Ulp2 fusions, R. Kawasumi and all lab members for discussions.

\section{FUNDING}

This work was supported by the Italian Association for Cancer Research (IG 18976, IG 23710), and European Research Council (Consolidator Grant 682190) grants to D.B., EMBO long-term fellowship (ALTF 561-2014) and an AIRC/Marie Curie Actions COFUND iCARE fellowship to I.P. Funding for open access charge: European Research Council (Consolidator Grant 682190).

\section{CONFLICT OF INTEREST}

None declared. 


\section{REFERENCES}

1. Flotho, A. and Melchior, F. (2013) Sumoylation: a regulatory protein modification in health and disease. Annu Rev Biochem, 82, 357-385.

2. Psakhye, I. and Jentsch, S. (2012) Protein group modification and synergy in the SUMO pathway as exemplified in DNA repair. Cell, 151, 807-820.

3. Jentsch, S. and Psakhye, I. (2013) Control of nuclear activities by substrateselective and protein-group SUMOylation. Annu Rev Genet, 47, 167-186.

4. Hickey, C.M., Wilson, N.R. and Hochstrasser, M. (2012) Function and regulation of SUMO proteases. Nat Rev Mol Cell Biol, 13, 755-766.

5. Sriramachandran, A.M. and Dohmen, R.J. (2014) SUMO-targeted ubiquitin ligases. Biochim Biophys Acta, 1843, 75-85.

6. Psakhye, I., Castellucci, F. and Branzei, D. (2019) SUMO-chain-regulated proteasomal degradation timing exemplified in DNA replication initiation. Mol Cell, 76, 632-645.

7. Nasmyth, K. and Haering, C.H. (2005) The structure and function of SMC and kleisin complexes. Annu Rev Biochem, 74, 595-648.

8. Yatskevich, S., Rhodes, J. and Nasmyth, K. (2019) Organization of chromosomal DNA by SMC complexes. Annu Rev Genet, 53, 445-482.

9. Almedawar, S., Colomina, N., Bermudez-Lopez, M., Pocino-Merino, I. and Torres-Rosell, J. (2012) A SUMO-dependent step during establishment of sister chromatid cohesion. Curr Biol, 22, 1576-1581.

10. McAleenan, A., Cordon-Preciado, V., Clemente-Blanco, A., Liu, I.C., Sen, N., Leonard, J., Jarmuz, A. and Aragon, L. (2012) SUMOylation of the alpha-kleisin subunit of cohesin is required for DNA damage-induced cohesion. Curr Biol, 22, 15641575 .

11. D'Ambrosio, L.M. and Lavoie, B.D. (2014) Pds5 prevents the polySUMOdependent separation of sister chromatids. Curr Biol, 24, 361-371. 
12. Wagner, K., Kunz, K., Piller, T., Tascher, G., Holper, S., Stehmeier, P., KeitenSchmitz, J., Schick, M., Keller, U. and Muller, S. (2019) The SUMO isopeptidase SENP6 functions as a rheostat of chromatin residency in genome maintenance and chromosome dynamics. Cell Rep, 29, 480-494.

13. Janke, C., Magiera, M.M., Rathfelder, N., Taxis, C., Reber, S., Maekawa, H., Moreno-Borchart, A., Doenges, G., Schwob, E., Schiebel, E. et al. (2004) A versatile toolbox for PCR-based tagging of yeast genes: new fluorescent proteins, more markers and promoter substitution cassettes. Yeast, 21, 947-962.

14. Stead, K., Aguilar, C., Hartman, T., Drexel, M., Meluh, P. and Guacci, V. (2003) Pds5p regulates the maintenance of sister chromatid cohesion and is sumoylated to promote the dissolution of cohesion. J Cell Biol, 163, 729-741.

15. Sherman, F. (1991) Getting started with yeast. Method Enzymol, 194, 3-21.

16. Psakhye, I. and Jentsch, S. (2016) Identification of substrates of protein-group SUMOylation. Methods Mol Biol, 1475, 219-231.

17. Borges, V., Lehane, C., Lopez-Serra, L., Flynn, H., Skehel, M., Rolef BenShahar, T. and Uhlmann, F. (2010) Hos1 deacetylates Smc3 to close the cohesin acetylation cycle. Mol Cell, 39, 677-688.

18. Mann, M. (2006) Functional and quantitative proteomics using SILAC. Nat Rev Mol Cell Biol, 7, 952-958.

19. Cox, J. and Mann, M. (2008) MaxQuant enables high peptide identification rates, individualized p.p.b.-range mass accuracies and proteome-wide protein quantification. Nat Biotechnol, 26, 1367-1372.

20. Eckhoff, J. and Dohmen, R.J. (2015) In vitro studies reveal a sequential mode of chain processing by the yeast SUMO (Small Ubiquitin-related Modifier)-specific protease Ulp2. J Biol Chem, 290, 12268-12281.

21. Wu, N., Kong, X., Ji, Z., Zeng, W., Potts, P.R., Yokomori, K. and Yu, H. (2012) Scc1 sumoylation by Mms21 promotes sister chromatid recombination through counteracting Wapl. Genes Dev, 26, 1473-1485. 
22. Takahashi, Y., Dulev, S., Liu, X., Hiller, N.J., Zhao, X. and Strunnikov, A. (2008) Cooperation of sumoylated chromosomal proteins in rDNA maintenance. PLoS Genet, 4, e1000215.

23. Potts, P.R., Porteus, M.H. and Yu, H. (2006) Human SMC5/6 complex promotes sister chromatid homologous recombination by recruiting the SMC1/3 cohesin complex to double-strand breaks. EMBO J, 25, 3377-3388.

24. Elmore, Z.C., Donaher, M., Matson, B.C., Murphy, H., Westerbeck, J.W. and Kerscher, O. (2011) Sumo-dependent substrate targeting of the SUMO protease Ulp1. BMC Biol, 9, 74 .

25. Losada, A. (2014) Cohesin in cancer: chromosome segregation and beyond. Nat Rev Cancer, 14, 389-393.

26. Dorsett, D. (2011) Cohesin: genomic insights into controlling gene transcription and development. Curr Opin Genet Dev, 21, 199-206.

27. Nasmyth, K. and Haering, C.H. (2009) Cohesin: its roles and mechanisms. Annu Rev Genet, 43, 525-558.

28. Hartman, T., Stead, K., Koshland, D. and Guacci, V. (2000) Pds5p is an essential chromosomal protein required for both sister chromatid cohesion and condensation in Saccharomyces cerevisiae. J Cell Biol, 151, 613-626.

29. Panizza, S., Tanaka, T., Hochwagen, A., Eisenhaber, F. and Nasmyth, K. (2000) Pds5 cooperates with cohesin in maintaining sister chromatid cohesion. Curr Biol, 10, 1557-1564.

30. Tong, K. and Skibbens, R.V. (2015) Pds5 regulators segregate cohesion and condensation pathways in Saccharomyces cerevisiae. Proc Natl Acad Sci U S A, 112, 7021-7026.

31. Kubota, T., Nishimura, K., Kanemaki, M.T. and Donaldson, A.D. (2013) The Elg1 replication factor C-like complex functions in PCNA unloading during DNA replication. Mol Cell, 50, 273-280. 
32. Kueng, S., Hegemann, B., Peters, B.H., Lipp, J.J., Schleiffer, A., Mechtler, K. and Peters, J.M. (2006) Wapl controls the dynamic association of cohesin with chromatin. Cell, 127, 955-967.

33. Maradeo, M.E. and Skibbens, R.V. (2010) Replication factor C complexes play unique pro- and anti-establishment roles in sister chromatid cohesion. PloS one, $\mathbf{5}$, e15381.

34. Li, Y., Muir, K.W., Bowler, M.W., Metz, J., Haering, C.H. and Panne, D. (2018) Structural basis for Scc3-dependent cohesin recruitment to chromatin. eLife, 7, e38356.

35. Ciosk, R., Shirayama, M., Shevchenko, A., Tanaka, T., Toth, A. and Nasmyth, K. (2000) Cohesin's binding to chromosomes depends on a separate complex consisting of Scc2 and Scc4 proteins. Mol Cell, 5, 243-254.

36. Unal, E., Heidinger-Pauli, J.M., Kim, W., Guacci, V., Onn, I., Gygi, S.P. and Koshland, D.E. (2008) A molecular determinant for the establishment of sister chromatid cohesion. Science, 321, 566-569.

37. Ben-Shahar, T.R., Heeger, S., Lehane, C., East, P., Flynn, H., Skehel, M. and Uhlmann, F. (2008) Eco1-dependent cohesin acetylation during establishment of sister chromatid cohesion. Science, 321, 563-566.

38. Noble, D., Kenna, M.A., Dix, M., Skibbens, R.V., Unal, E. and Guacci, V. (2006) Intersection between the regulators of sister chromatid cohesion establishment and maintenance in budding yeast indicates a multi-step mechanism. Cell Cycle, 5, 2528-2536.

39. Ladurner, R., Bhaskara, V., Huis in 't Veld, P.J., Davidson, I.F., Kreidl, E., Petzold, G. and Peters, J.M. (2014) Cohesin's ATPase activity couples cohesin loading onto DNA with Smc3 acetylation. Curr Biol, 24, 2228-2237.

40. Petela, N.J., Gligoris, T.G., Metson, J., Lee, B.G., Voulgaris, M., Hu, B., Kikuchi, S., Chapard, C., Chen, W., Rajendra, E. et al. (2018) Scc2 is a potent activator of cohesin's ATPase that promotes loading by binding Scc1 without Pds5. Mol Cell, 70, 1134-1148. 
41. Li, S.J. and Hochstrasser, M. (2000) The yeast ULP2 (SMT4) gene encodes a novel protease specific for the ubiquitin-like Smt3 protein. Mol Cell Biol, 20, 23672377.

42. Li, S.J. and Hochstrasser, M. (1999) A new protease required for cell-cycle progression in yeast. Nature, 398, 246-251.

43. Li, S.J. and Hochstrasser, M. (2003) The Ulp1 SUMO isopeptidase: distinct domains required for viability, nuclear envelope localization, and substrate specificity. J Cell Biol, 160, 1069-1081.

44. Panse, V.G., Kuster, B., Gerstberger, T. and Hurt, E. (2003) Unconventional tethering of Ulp1 to the transport channel of the nuclear pore complex by karyopherins. Nat Cell Biol, 5, 21-27.

45. Mossessova, E. and Lima, C.D. (2000) Ulp1-SUMO crystal structure and genetic analysis reveal conserved interactions and a regulatory element essential for cell growth in yeast. Mol Cell, 5, 865-876.

46. Liang, J., Singh, N., Carlson, C.R., Albuquerque, C.P., Corbett, K.D. and Zhou, H. (2017) Recruitment of a SUMO isopeptidase to rDNA stabilizes silencing complexes by opposing SUMO targeted ubiquitin ligase activity. Genes Dev, 31, 802815.

47. Suhandynata, R.T., Quan, Y., Yang, Y., Yuan, W.T., Albuquerque, C.P. and Zhou, H. (2019) Recruitment of the Ulp2 protease to the inner kinetochore prevents its hyper-sumoylation to ensure accurate chromosome segregation. PLoS Genet, 15, e1008477.

48. Xu, X. and Yanagida, M. (2019) Suppressor screening reveals common kleisinhinge interaction in condensin and cohesin, but different modes of regulation. Proc Natl Acad Sci U S A, 116, 10889-10898.

49. Robellet, X., Fauque, L., Legros, P., Mollereau, E., Janczarski, S., Parrinello, H., Desvignes, J.P., Thevenin, M. and Bernard, P. (2014) A genetic screen for functional partners of condensin in fission yeast. G3 (Bethesda), 4, 373-381. 
50. Strunnikov, A.V., Aravind, L. and Koonin, E.V. (2001) Saccharomyces cerevisiae SMT4 encodes an evolutionarily conserved protease with a role in chromosome condensation regulation. Genetics, 158, 95-107.

51. D'Amours, D., Stegmeier, F. and Amon, A. (2004) Cdc14 and condensin control the dissolution of cohesin-independent chromosome linkages at repeated DNA. Cell, 117, 455-469.

52. Nishimura, K., Fukagawa, T., Takisawa, H., Kakimoto, T. and Kanemaki, M. (2009) An auxin-based degron system for the rapid depletion of proteins in nonplant cells. Nat Methods, 6, 917-922.

53. Menolfi, D., Delamarre, A., Lengronne, A., Pasero, P. and Branzei, D. (2015) Essential roles of the Smc5/6 complex in replication through natural pausing sites and endogenous DNA damage tolerance. Mol Cell, 60, 835-846.

54. Mullen, J.R., Das, M. and Brill, S.J. (2011) Genetic evidence that polysumoylation bypasses the need for a SUMO-targeted Ub ligase. Genetics, 187, 7387.

55. Zhao, X. and Blobel, G. (2005) A SUMO ligase is part of a nuclear multiprotein complex that affects DNA repair and chromosomal organization. Proc Natl Acad Sci U $S A, \mathbf{1 0 2}, 4777-4782$.

56. Bustard, D.E., Ball, L.G. and Cobb, J.A. (2016) Non-Smc element 5 (Nse5) of the Smc5/6 complex interacts with SUMO pathway components. Biol Open, 5, 777785.

57. Branzei, D., Sollier, J., Liberi, G., Zhao, X., Maeda, D., Seki, M., Enomoto, T., Ohta, K. and Foiani, M. (2006) Ubc9- and mms21-mediated sumoylation counteracts recombinogenic events at damaged replication forks. Cell, 127, 509-522.

58. Sollier, J., Driscoll, R., Castellucci, F., Foiani, M., Jackson, S.P. and Branzei, D. (2009) The Saccharomyces cerevisiae Esc2 and Smc5-6 proteins promote sister chromatid junction-mediated intra-S repair. Mol Biol Cell, 20, 1671-1682.

59. Baldwin, M.L., Julius, J.A., Tang, X., Wang, Y. and Bachant, J. (2009) The yeast SUMO isopeptidase Smt4/Ulp2 and the polo kinase Cdc5 act in an opposing 
fashion to regulate sumoylation in mitosis and cohesion at centromeres. Cell Cycle, $\mathbf{8}$, 3406-3419.

60. Alexandru, G., Uhlmann, F., Mechtler, K., Poupart, M.A. and Nasmyth, K. (2001) Phosphorylation of the cohesin subunit Scc1 by Polo/Cdc5 kinase regulates sister chromatid separation in yeast. Cell, 105, 459-472.

61. Shi, Z., Gao, H., Bai, X.C. and Yu, H. (2020) Cryo-EM structure of the human cohesin-NIPBL-DNA complex. Science, 368, 1454-1459.

62. Hons, M.T., Huis In 't Veld, P.J., Kaesler, J., Rombaut, P., Schleiffer, A., Herzog, F., Stark, H. and Peters, J.M. (2016) Topology and structure of an engineered human cohesin complex bound to Pds5B. Nat Commun, 7, 12523.

63. Moldovan, G.L., Pfander, B. and Jentsch, S. (2006) PCNA controls establishment of sister chromatid cohesion during S phase. Mol Cell, 23, 723-732.

64. Srinivasan, M., Scheinost, J.C., Petela, N.J., Gligoris, T.G., Wissler, M., Ogushi, S., Collier, J.E., Voulgaris, M., Kurze, A., Chan, K.L. et al. (2018) The cohesin ring uses its hinge to organize DNA using non-topological as well as topological mechanisms. Cell, 173, 1508-1519.

65. Dantuma, N.P. and Hoppe, T. (2012) Growing sphere of influence: Cdc48/p97 orchestrates ubiquitin-dependent extraction from chromatin. Trends Cell Biol, 22, 483491.

66. Franz, A., Ackermann, L. and Hoppe, T. (2016) Ring of change: CDC48/p97 drives protein dynamics at chromatin. Front Genet, 7, 73.

67. Frattini, C., Villa-Hernandez, S., Pellicano, G., Jossen, R., Katou, Y., Shirahige, K. and Bermejo, R. (2017) Cohesin ubiquitylation and mobilization facilitate stalled replication fork dynamics. Mol Cell, 68, 758-772.

68. Thattikota, Y., Tollis, S., Palou, R., Vinet, J., Tyers, M. and D'Amours, D. (2018) Cdc48/VCP promotes chromosome morphogenesis by releasing condensin from self-entrapment in chromatin. Mol Cell, 69, 664-676. 


\section{FIGURE LEGENDS}

\section{Figure 1. SUMO chains promote the turnover of SUMOylated SMC complex subunits in the absence of Ulp2 SUMO protease}

Outline of SILAC experiment performed to detect degradation-prone SUMOylated substrates that decrease in abundance in a SUMO-chain-dependent manner in ulp2 $\Delta$ cim3-1 cells (left). SILAC ratios for 726 quantified proteins plotted against the sum of the relevant peptide intensities (right). SUMOylated subunits of cohesin (red), condensin (blue) and the Smc5/6 complex (green) accumulate if instead of HisSUMO, which is able to form SUMO chains, a lysine-less SUMO variant (KRall) that cannot form lysinelinked SUMO chains is expressed as the only source of SUMO.

Figure 2. The essential role of cohesin-associated subunit Pds5 is to counteract

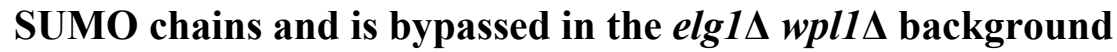

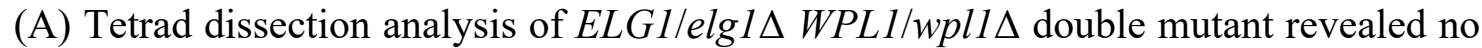
negative genetic interaction compared to WT.

(B) Lethality of $p d s 5 \Delta$ cells is suppressed by SUMO mutant $s m t 3-K R a l l$ that cannot form lysine-linked SUMO chains.

(C) Lethality of $p d s 5 \Delta$ cells is bypassed in $\operatorname{elg} 1 \Delta w p l 1 \Delta$ background.

(D) Smc3 lysine K112, K113 acetylation is decreased in $p d s 5 \Delta$ cells, lethality of which

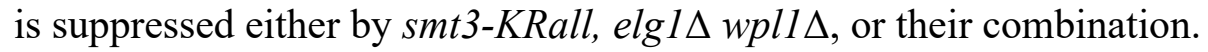

(E) $p d s 5 \Delta$ elg $1 \Delta w p l 1 \Delta$ cells are slow-growth and exhibit sensitivity to temperature, $\mathrm{HU}$, MMS, CPT and benomyl. Spotting of 1:7 serial dilutions on YPD plates at indicated temperatures and drug concentrations. 
(F) Viability of $p d s 5 \Delta$ elgl $1 \Delta$ wpll $\Delta$ cells depends on ULP2. Upon transcriptional shutoff of $U L P 2$ expressed from inducible $G A L$ promoter $p d s 5 \Delta$ elgl $1 \Delta p l 1 \Delta$ cells die.

(G) Lethality of $p d s 5 \Delta \operatorname{elg} 1 \Delta$ wpl1 $\Delta$ ulp 2 cells is bypassed by the spontaneous suppressor of $u l p 2 \Delta$ phenotypes denoted $u l p 1-C 376$ that is no longer tethered to the nuclear pores and is able to deSUMOylate Ulp2 substrates in the nucleoplasm.

Figure 3. Loss of Pds5 triggers SUMOylation of cohesin's kleisin Scc1 that is counteracted by Ulp2

(A) Loss of Pds5 triggers SUMOylation of cohesin's kleisin Scc1 and leads to the reduction of its protein levels as well as of Smc3 lysine K112, K113 acetylation levels. Denaturing Ni-NTA pull-down (Ni PD) was performed to isolate His SUMO conjugates from cells expressing C-terminally 6HA-tagged Scc1 at endogenous levels and Nterminally 3HA-tagged Pds5 under the control of inducible GAL promoter. Cells were collected at the indicated time after shift from galactose- to glucose-containing media. Ni PD efficiency was assayed using anti-Smt3 antibody and Ponceau S staining. Pgk1 served as loading control.

(B) Expression of a lysine-less SUMO variant KRall that cannot form lysine-linked SUMO chains instead of His SUMO results in the accumulation of monoSUMOylated Scc1 species even prior to PDS5 shut-off. Loss of Ulp2 leads to further increase in Scc1 multiSUMOylation.

Figure 4. Fusion of Ulp2 to cohesin's kleisin Scc1 supports viability in the absence of Pds5 and protects cohesin from turnover

(A) Schematic representation of Scc1-Ulp2 fusions used in this study, fusion is the only source of cohesin's kleisin in the cell. Scc1 depicted with its binding regions to other 
cohesin subunits and separase cleavage sites. Ulp2 fragment (aa 1-734) is fused Cterminally to endogenous Scc1 without a linker. The N-terminus of Ulp2 harbors 5 SIMs (colored blue) necessary for binding to SUMO chains and is followed by the protease domain; C-terminus (aa 735-1034) is replaced with 6HA tag. Catalytically-active Ulp2 fusion to Scc1 is denoted scc1-ulp2 ${ }_{734}-6 H A$, catalytically-dead scc1-ulp2 ${ }_{734}-C 624 S-6 H A$ carries point mutation in active site, scc1-ulp2 ${ }_{734-s i m-6 H A}$ has all N-terminal SIMs mutated loosing ability to recognize SUMO chains.

(B) Catalytically-active scc1-ulp2 ${ }_{734}-6 H A$ fusion supports viability upon PDS5 shut-off, whereas scc1-ulp2 ${ }_{734}-C 624 S-6 H A$ and scc1-ulp2 ${ }_{734-s i m-6 H A}$ fusions fail to do so three days post spotting.

(C) Catalytically-active $s c c 1-u l p 2{ }_{734}-6 H A$ fusion supports viability of $p d s 5 \Delta$ elg $1 \Delta$ wpll $\triangle$ cells upon ULP2 shut-off, whereas catalytically-dead scc1-ulp2 ${ }_{734}-C 624 S-6 H A$ fusion does not.

(D-E) Upon $U L P 2$ shut-off in $\operatorname{elg} 1 \Delta w p l 1 \Delta p d s 5 \Delta$ background the protein levels of the catalytically-dead Ulp2 fusion to Scc1 (Scc1-ulp2 $\left.{ }_{734}-\mathrm{C} 624 \mathrm{~S}-6 \mathrm{HA}\right)$ decrease (D), whereas catalytically-active Scc1-ulp2 $734-6 \mathrm{HA}$ fusion remains stable (E).

Figure 5. Ulp2 fusion to cohesin's kleisin Scc1 keeps it in monoSUMOylated state and protects from SUMO-chain-targeted turnover

(A-B) Catalytically-active scc1-ulp2 ${ }_{734}-6 H A$ fusion maintains its protein and monoSUMOylation levels as well as Smc3 lysine K112, K113 acetylation levels upon $U L P 2$ shut-off in $p d s 5 \Delta$ elg $1 \Delta$ wpll $\Delta$ cells, whereas catalytically-dead scc1-ulp2 ${ }_{734}-$ C624S-6HA fusion does not. Ni PD was performed to isolate ${ }^{H i s}$ SUMO conjugates from cells expressing either scc1-ulp2 ${ }_{734}-6 H A$ (A) or scc1-ulp2 ${ }_{734}-C 624 S-6 H A(\mathrm{~B})$ and ULP2 under the control of inducible GAL promoter. Ni PD efficiency was assayed using anti- 
Smt3 antibody and Ponceau S staining. Pgk1 served as loading control. High molecular weight (HMW) polySUMO conjugates accumulate in Input upon ULP2 shut-off.

Figure 6. Condensin is protected by Ulp2 against SUMO-chain-mediated turnover

(A) Temperature sensitive smc2-8 mutant exhibits synthetic sick/lethal genetic interaction with $u l p 2 \Delta$ at permissive temperatures for $s m c 2-8$ cells. Spotting of 1:7 serial dilutions on YPD plates at indicated temperatures.

(B-C) Synthetic lethality of $s m c 2-8$ ulp $2 \Delta$ cells at permissive temperatures for $s m c 2-8$ single mutant is suppressed if a lysine-less SUMO variant (smt3-KRall) that cannot form SUMO chains is expressed as the only source of SUMO (B) or if STUbL subunit Slx5 is deleted (C).

(D) Levels of monoSUMOylated condensin's kleisin Brn1 are reduced in the absence of Ulp2 compared to WT, while expression of a lysine-less SUMO variant KRall restores them. Ni PD was performed to isolate SUMO conjugates from cells expressing C-terminally 6HA-tagged Brn1. Ni PD efficiency was assayed using anti-Smt3 antibody and Ponceau S staining. Pgk1 served as loading control.

Figure 7. The Smc5/6 complex is protected by Ulp2 against SUMO-chain-mediated turnover

(A) Temperature sensitive smc6-56 mutant exhibits synthetic sick/lethal genetic interaction with $u l p 2 \Delta$ at permissive temperatures for smc6-56 cells.

(B) Synthetic lethality of $s m c 6-56 u l p 2 \Delta$ cells at permissive temperatures for $s m c 6-56$ single mutant is suppressed by deletion of the SUMO ligase Siz2.

(C) Levels of monoSUMOylated Smc5/6 complex kleisin Nse4 are reduced in the absence of Ulp2 compared to WT and increased upon Slx5 deletion. Ni PD was 
performed to isolate ${ }^{H i s}$ SUMO conjugates from cells expressing C-terminally 6HAtagged Nse4. Ni PD efficiency was assayed using anti-Smt3 antibody and Ponceau S staining. Pgk1 served as loading control.

(D) monoSUMOylated Nse4 species are not degradation-prone as they are not accumulating in the proteasome-defective mutant cim3-1 background.

(E) Expression of a chainless SUMO variant KRall leads to the accumulation of monoSUMOylated Nse4 species both in WT and $u l p 2 \Delta$ cells. 
bioRxiv preprint doi: https://doi.org/10.1101/2020.11.13.381483; this version posted March 16,2021 . The copyright holder for this preprint (which was not certified by peer review) is the author/funder, who has granted bioRxiv a license to display the preprint in perpetuity. It is made available under aCC-BY-NC-ND 4.0 International license.

\section{Figure 1}
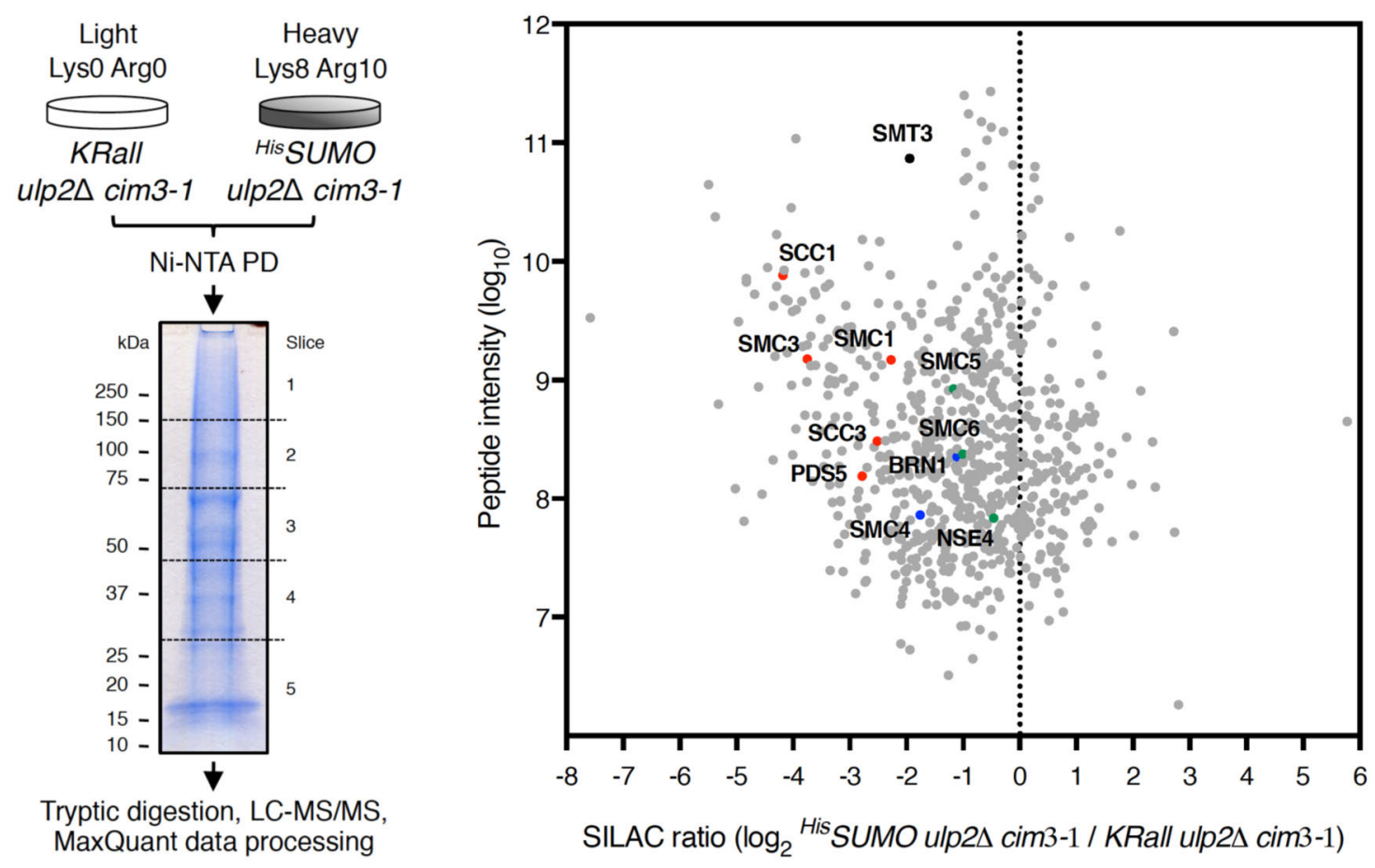


\section{Figure 2}

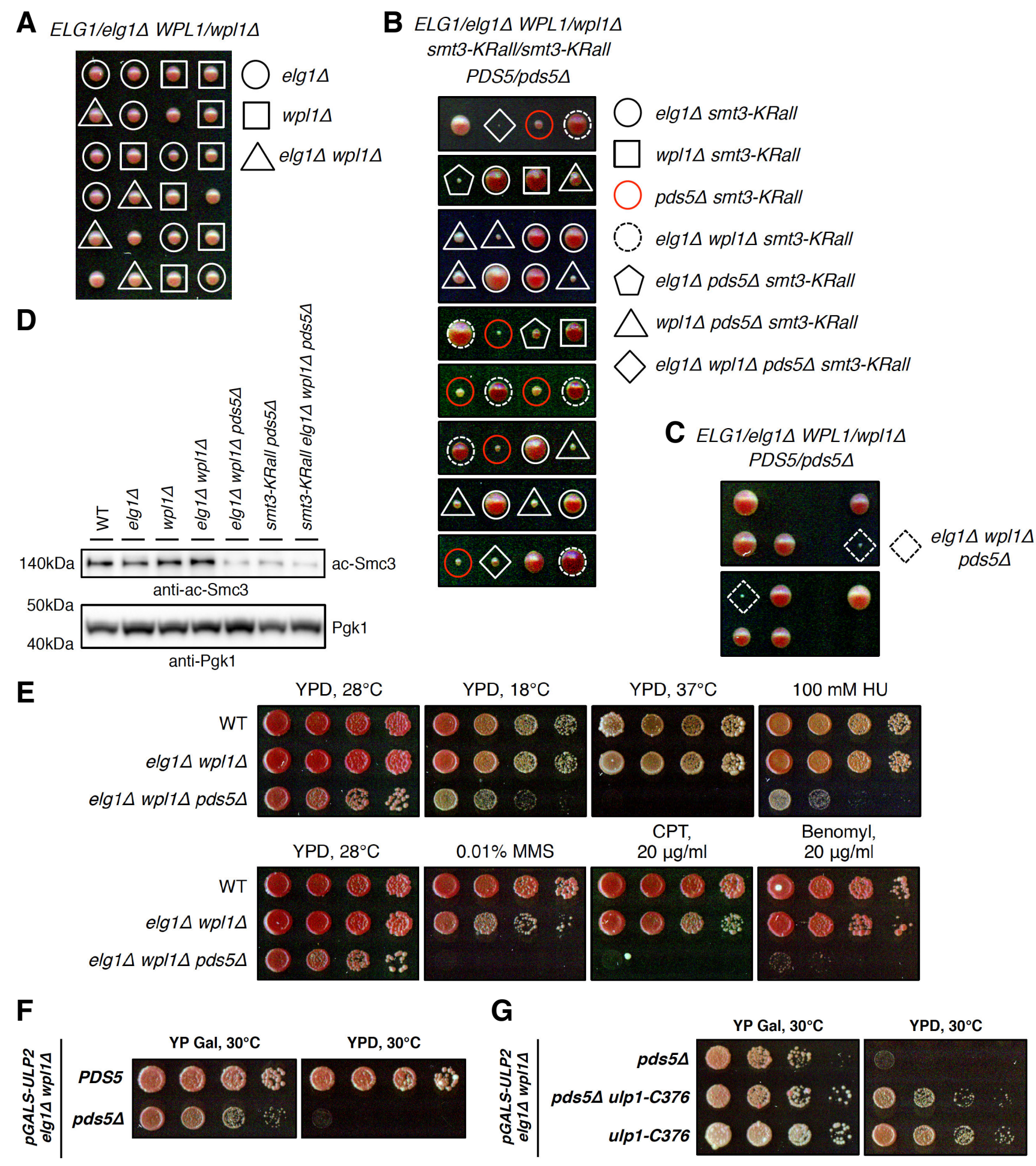


bioRxiv preprint doi: https://doi.org/10.1101/2020.11.13.381483; this version posted March 16, 2021. The copyright holder for this preprint (which was not certified by peer review) is the author/funder, who has granted bioRxiv a license to display the preprint in perpetuity. It is made available under aCC-BY-NC-ND 4.0 International license.

\section{Figure 3}

A

pGALS- ${ }^{3 H A} P d S 5$

Scc16HA HISSUMO

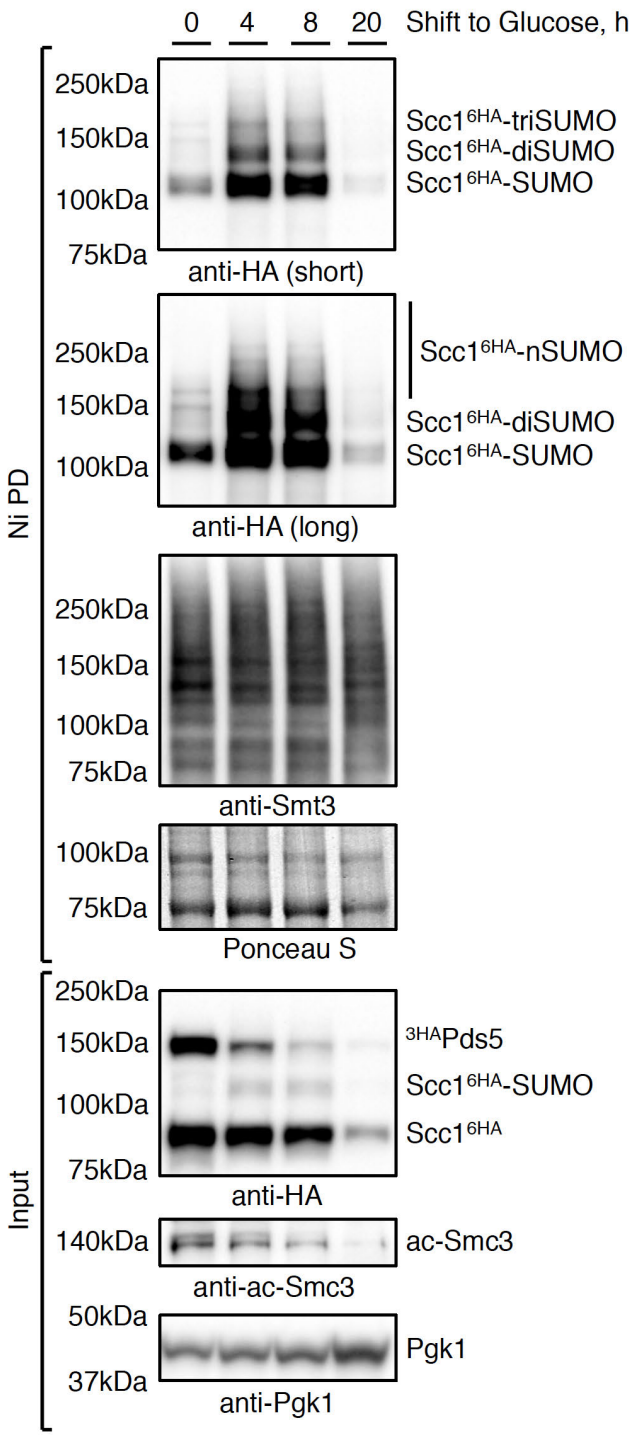

B

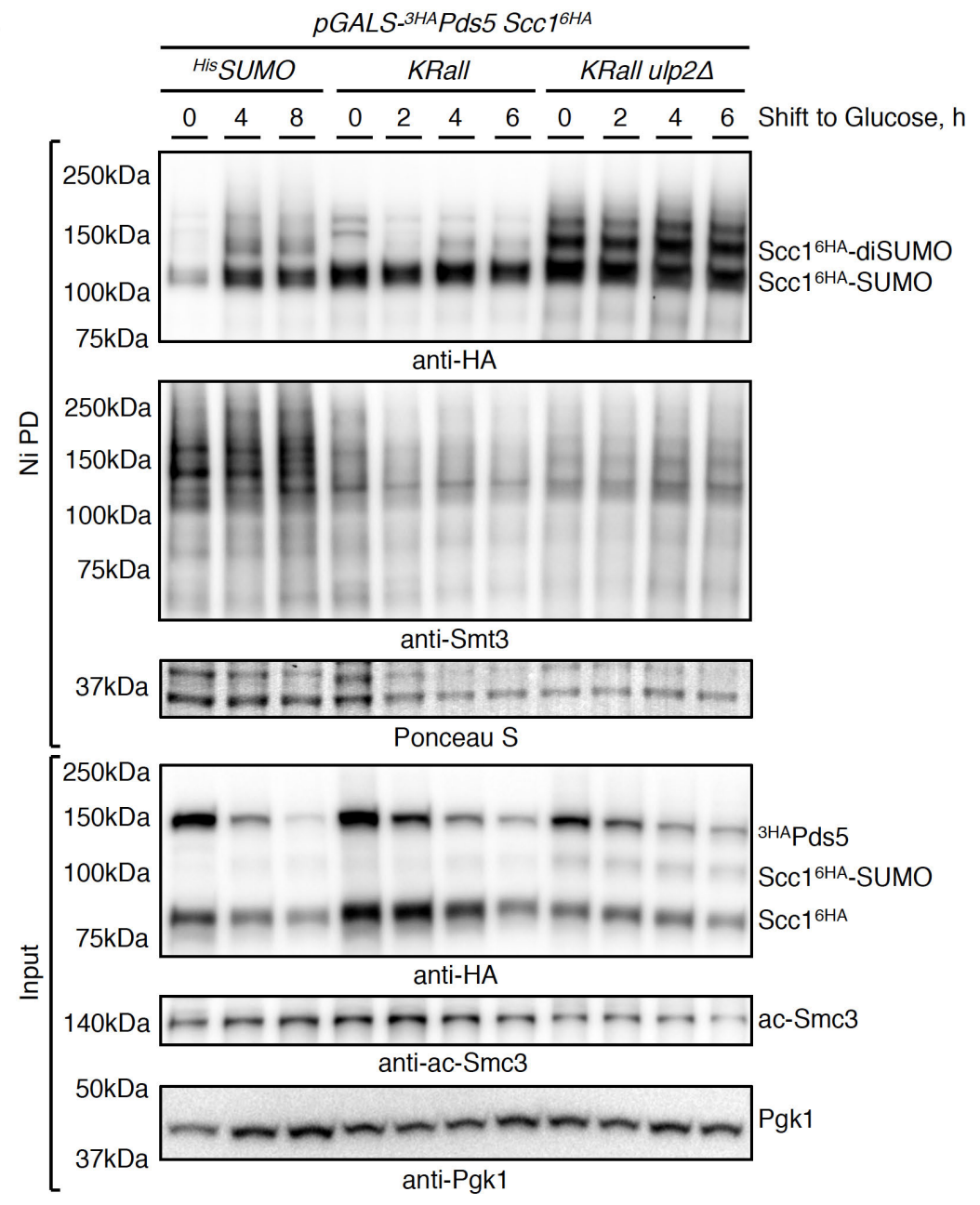


bioRxiv preprint doi: https://doi.org/10.1101/2020.11.13.381483; this version posted March 16, 2021. The copyright holder for this preprint (which was not certified by peer review) is the author/funder, who has granted bioRxiv a license to display the preprint in perpetuity. It is made available under aCC-BY-NC-ND 4.0 International license.

\section{Figure 4}

A
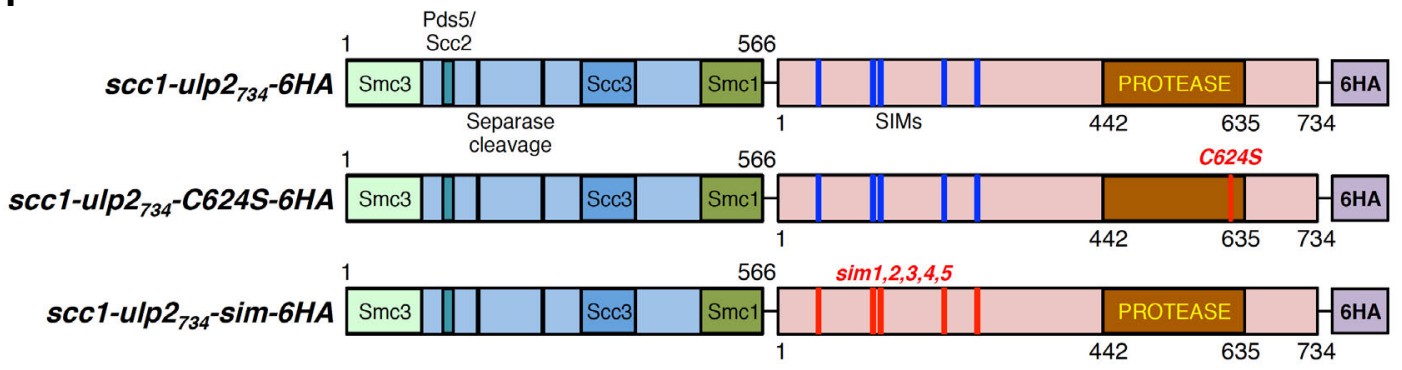

B

C YP Gal, $3^{\circ} \mathrm{C} \quad \mathrm{YPD}, 30^{\circ} \mathrm{C}$

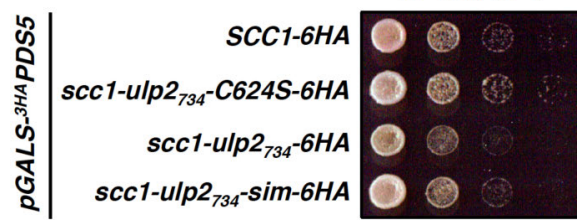

$00 \%$

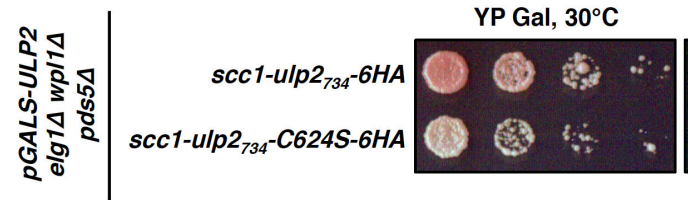

YPD, $30^{\circ} \mathrm{C}$

D

pGALS-ULP2

E

pGALS-ULP2

wpl1 $1 \Delta$ elg1 $1 \Delta d s 5 \Delta$

wpl1 $\triangle$ elg1 $\triangle$ pds $5 \Delta$

scc1-ulp2 ${ }_{734}-$ C624S-6HA

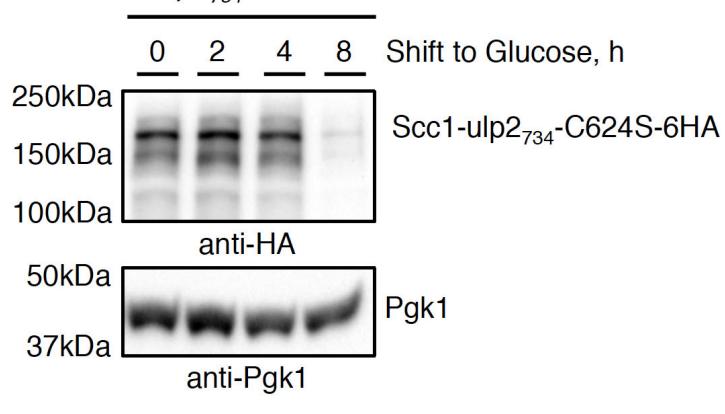


bioRxiv preprint doi: https://doi.org/10.1101/2020.11.13.381483; this version posted March 16,2021 . The copyright holder for this preprint (which was not certified by peer review) is the author/funder, who has granted bioRxiv a license to display the preprint in perpetuity. It is made available under aCC-BY-NC-ND 4.0 International license.

\section{Figure 5}

A

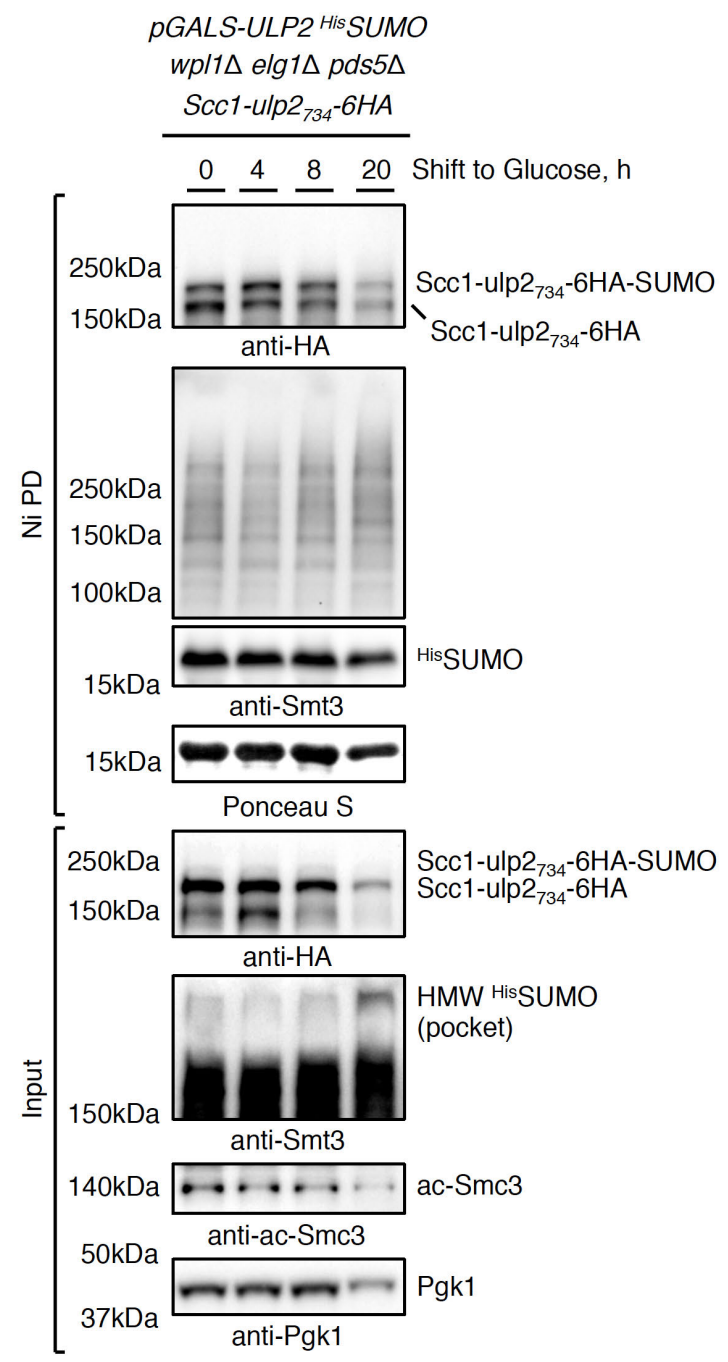

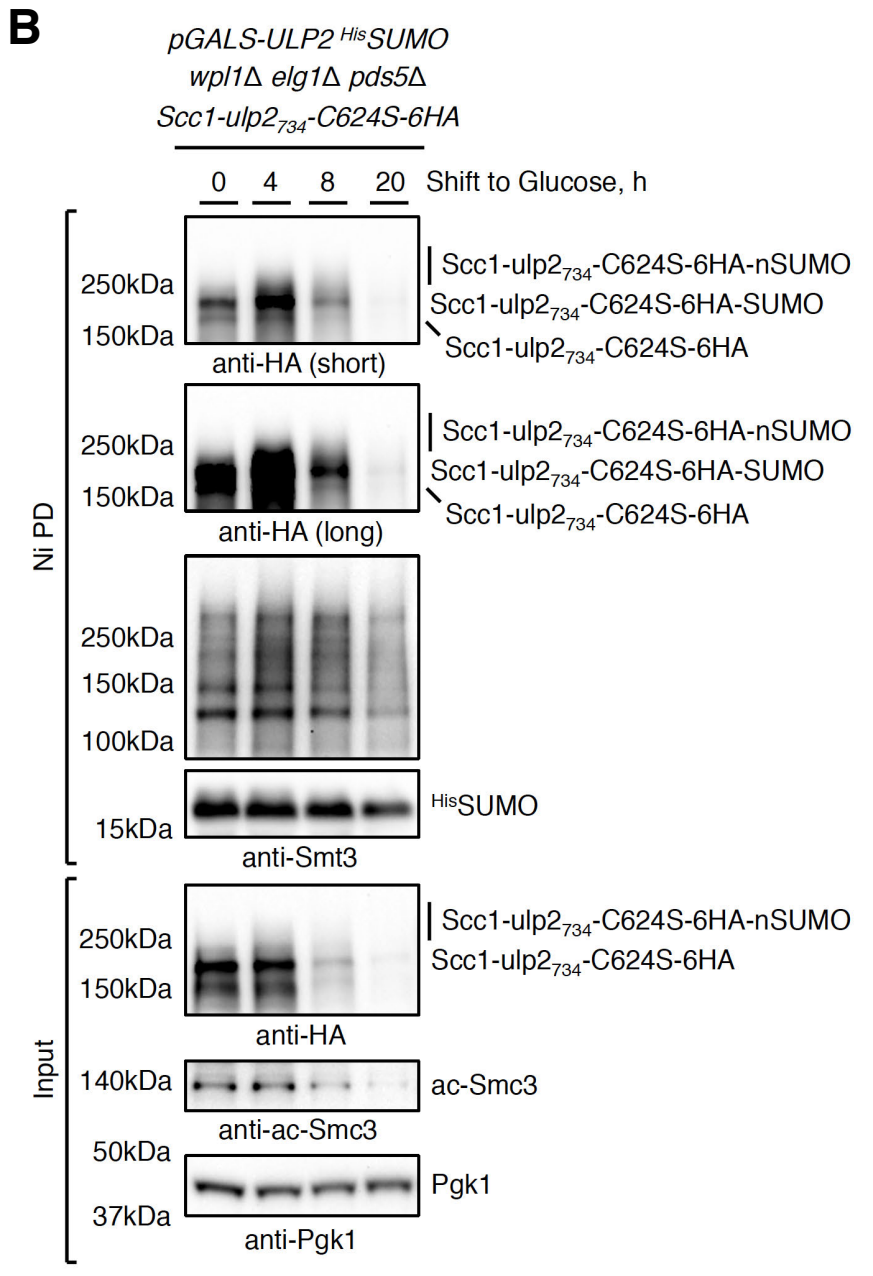


bioRxiv preprint doi: https://doi.org/10.1101/2020.11.13.381483; this version posted March 16, 2021. The copyright holder for this preprint

(which was not certified by peer review) is the author/funder, who has granted bioRxiv a license to display the preprint in perpetuity. It is made available under aCC-BY-NC-ND 4.0 International license.

\section{Figure 6}

A

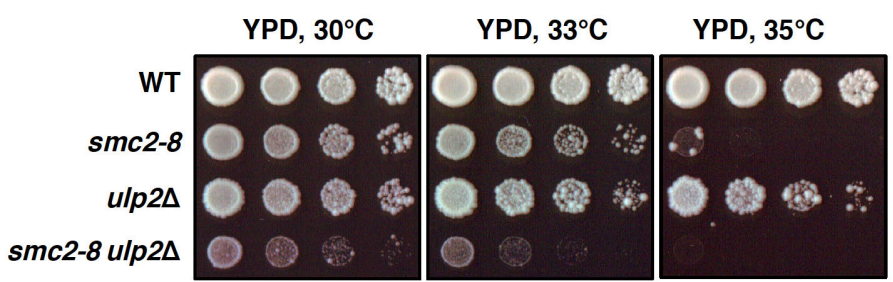

B

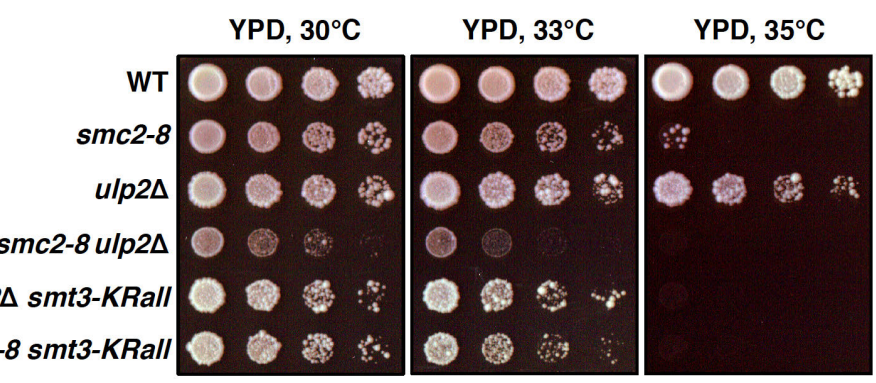

C

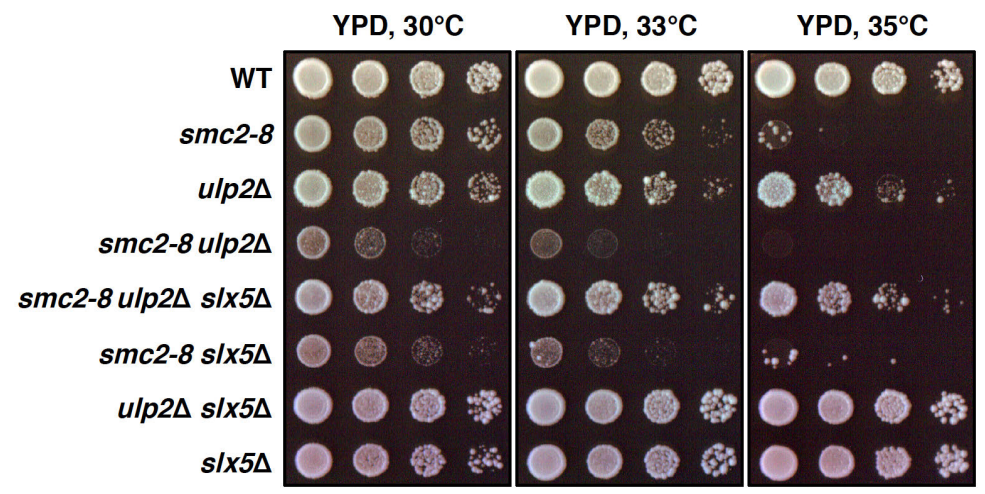

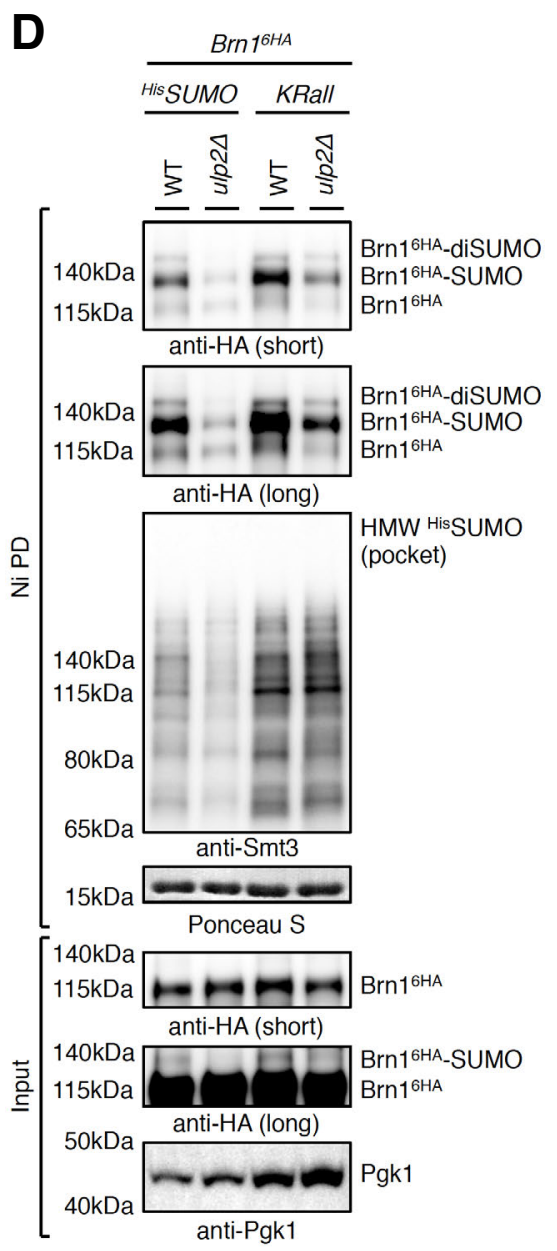


bioRxiv preprint doi: https://doi.org/10.1101/2020.11.13.381483; this version posted March 16, 2021. The copyright holder for this preprint

(which was not certified by peer review) is the author/funder, who has granted bioRxiv a license to display the preprint in perpetuity. It is made available under aCC-BY-NC-ND 4.0 International license.

\section{Figure 7}

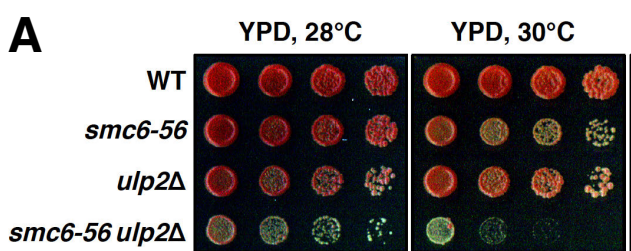

C

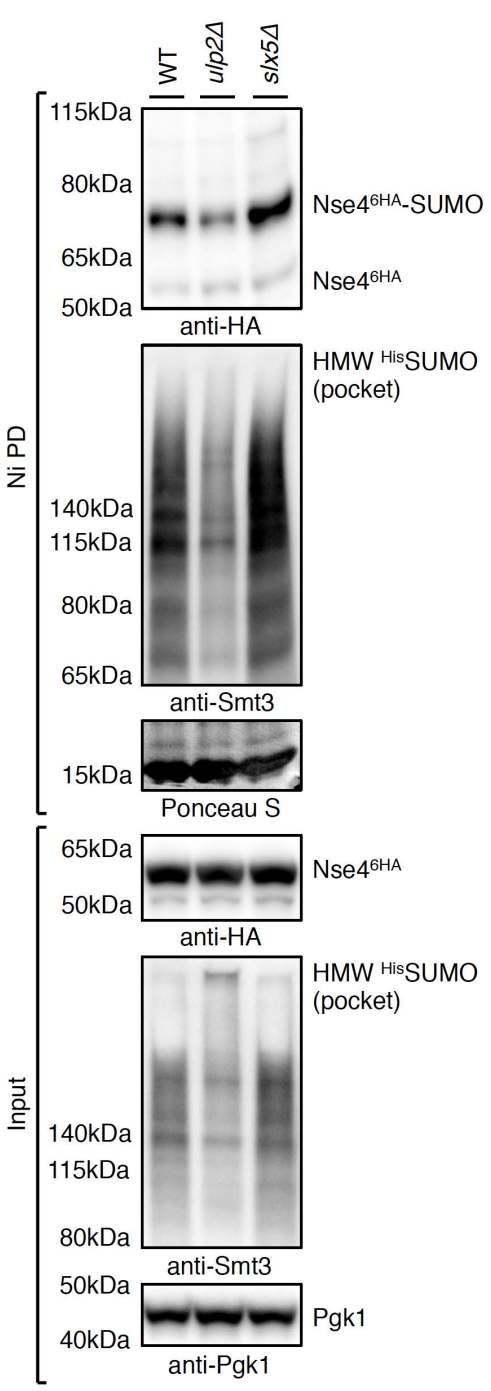

YPD, $37^{\circ} \mathrm{C} \quad \mathrm{B}$

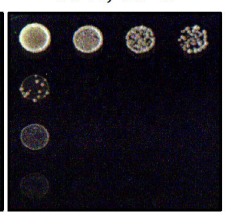

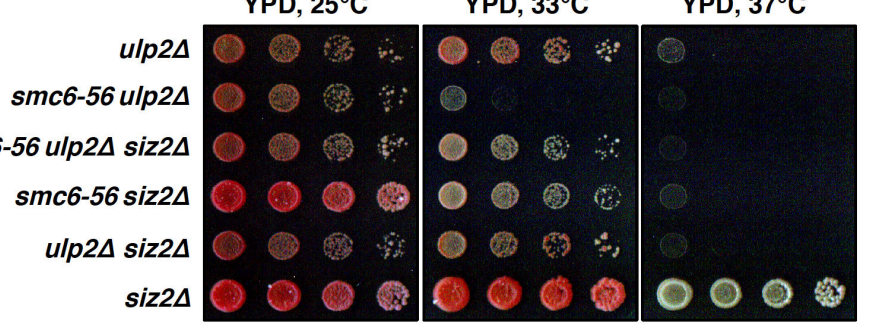

D

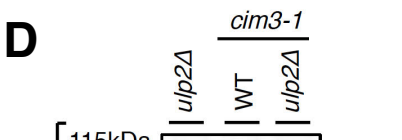

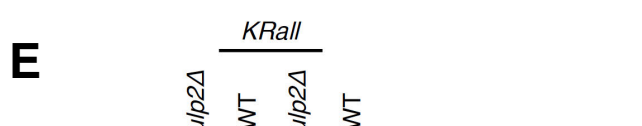
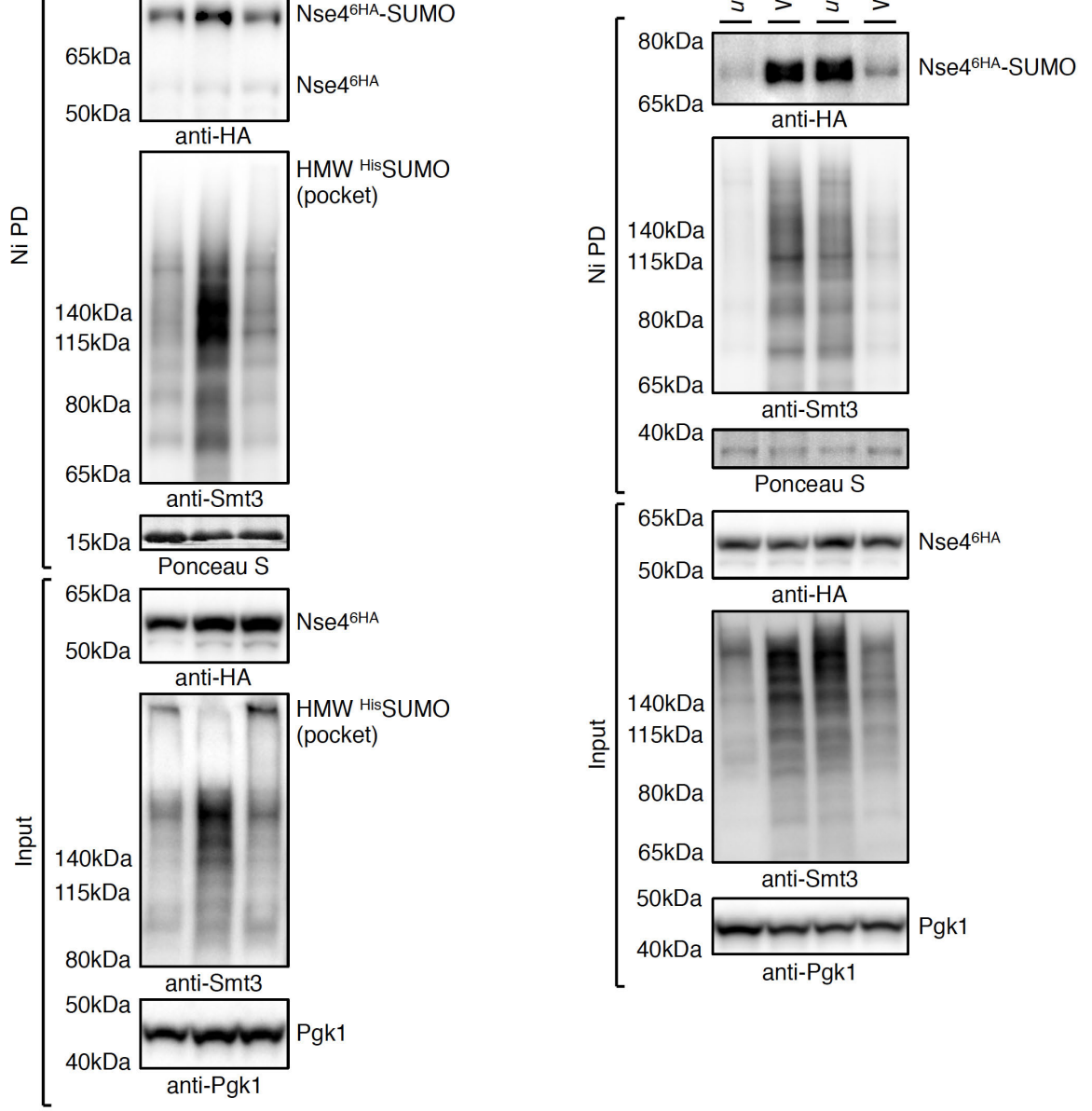